\title{
Genre et travail dans l'iconographie monétaire en circulation au Cameroun de 1945 à 2002
}

Gender and labour in monetary iconography of currency circulating in

Cameroon from 1945 to 2002

Joceline Clarisse Mafossi

\section{OpenEdition}

Journals

Édition électronique

URL : http://journals.openedition.org/itti/744

DOI : 10.4000/itti.744

Éditeur

Université de Poitiers

Référence électronique

Joceline Clarisse Mafossi, «Genre et travail dans l'iconographie monétaire en circulation au

Cameroun de 1945 à 2002 », Images du travail, travail des images [En ligne], 6-7 | 2019, mis en ligne le

01 février 2019, consulté le 14 avril 2021. URL : http://journals.openedition.org/itti/744 ; DOI : https:// doi.org/10.4000/itti.744

Ce document a été généré automatiquement le 14 avril 2021

Images du travail, travail des images 


\section{Genre et travail dans l'iconographie monétaire en circulation au Cameroun de 1945 à 2002}

Gender and labour in monetary iconography of currency circulating in

Cameroon from 1945 to 2002

Joceline Clarisse Mafossi

1 Les études portant sur le genre et le travail dans l'iconographie monétaire en général et des monnaies coloniales en particulier, ne sont pas les plus courantes, tant en numismatique, en archéologie, en sémiologie qu'en histoire. Cela se justifie par l'entrée tardive des images monétaires dans la catégorie d'objet d'étude (Schomas, 2011) et par les angles d'approche de ces images considérées comme un témoignage historique dans le temps et dans l'espace (Picard, 1991; Monpas, 2006). Ces «mass médias » (Caire, 2001) qui servent de canal de communication entre l'institution sociale et les populations (Perez, 1985) sont aussi considérés comme des images d'État que Michel Dupré appelle gracieusement « l'image populaire d'État 》 (Dupré, 2001). Les notions de propagande politique, de pouvoir, d'histoire, de progrès économique, de religion et de sécurité ont longtemps fait ombrage à des éléments socioculturels profondément ancrés dans ces minuscules icônes qui participent, d'une certaine manière, à la structuration et la construction d'un idéal social. C'est le cas des questions telles que le genre et le travail qui font l'objet de cette étude' ${ }^{1}$.

2 Les places occupées par les hommes et les femmes dans l'organisation sociale du travail se trouvent au cœur du concept du genre et varient d'une société à une autre. Le genre est ici conçu comme un rapport social de sexe, comme une entrée pour comprendre le fonctionnement d'une société et les valeurs qu'on accorde à chaque sexe. En effet, au sein de chaque peuple, le fait d'être homme ou d'être femme semble suffire pour définir le travail correspondant, et l'apport de chacun dans le développement (croissance économique, perpétuité de l'espèce). C'est le cas du Cameroun, pays d'Afrique centrale, qui, malgré sa diversité culturelle (plus de 200 ethnies), partage une considération plus ou moins commune de la répartition des tâches selon le sexe. Le concept de genre très 
développé et débattu en Occident est encore en éclosion en Afrique et particulièrement au Cameroun. Cela tient à la distance entre les valeurs culturelles traditionnelles existantes depuis des siècles et celles introduites par la colonisation et qui aurait influencé la hiérarchisation des tâches dans la société ${ }^{2}$. Cette dernière caractérise donc l'ensemble de l'iconographie des monnaies émises par la France au Cameroun entre 1945 et 2002.

3 L'iconographie monétaire, rarement définie par les chercheurs qui s'y sont intéressés, fait ici référence à l'ensemble des images inscrites sur les monnaies par gravure (monnaies métalliques) et impression (papier monnaie). Destinées au peuple, elles s'inscrivent dans une cellule de communication (Perez, 1985), qui n'est pas toujours transparente, manipulée par le pouvoir en place. On peut sommairement catégoriser deux types d'images monétaires: celles émises par l'institution d'un État autonome pour son territoire et celles émises par une puissance dans un territoire sur lequel il exerce une force, un pouvoir. C'est le cas des monnaies coloniales, créées avec les banques coloniales, qui ont été maintenues après les indépendances - cas du Franc CFA - pour répondre à certains besoins d'ordre économique et politique (Renaud, 1899). L'iconographie monétaire coloniale, puis postcoloniale dont il est question dans le cas spécifique de cette étude présente donc une réalité plus complexe.

4 En effet, l'action coloniale s'est accompagnée de plusieurs instruments au rang desquels on peut citer la monnaie. Au début, ces monnaies coloniales ont été créées pour permettre à chaque territoire d'affirmer sa personnalité (Reuter, 1950). Pourtant, saisis par leur large diffusion et leur utilité quotidienne obligatoire, les colonisateurs français les ont très tôt introduites en Afrique (Deganendji, 2006), non seulement comme marque d'autorité mais également comme support d'expression. Au même titre que les affiches coloniales, l'imagerie coloniale y est clairement exposée, développant plusieurs thèmes étroitement liés aux attentes et à la perception que la métropole avait de ses possessions d'une part, et de ce qu'elle voulait que la République retienne des multiples entreprises coloniales d'autre part (Blanchard, 2001). Ainsi, ces représentations sont accompagnées d'une construction du genre au sein de l'empire colonial destinée à la métropole : rentabilité à travers l'implication dans les divers travaux et privilèges en cas de besoin $d^{\prime}$ " affection $»^{3}$. Parmi les thèmes développés dans ce champ iconographique, le travail ou du moins, les fruits du travail sont au centre des représentations ${ }^{4}$. Après les indépendances, ces formes de représentations exotiques et de tradition occidentale ont suivi leur cours en épousant les nouvelles dynamiques sociopolitiques ancrées dans le néocolonialisme ${ }^{5}$. Le Cameroun, annexé par la France dès 1916 n'a pas échappé à cette pratique avec l'utilisation de la monnaie moderne au début de la colonisation allemande en 1891, puis l'adoption officielle du Franc CFA et son intégration à la Zone Franc ${ }^{6}$.

5 Après la Seconde Guerre mondiale, la France entreprend une stratégie de regroupement de son empire colonial en créant des zones monétaires. Le Franc CFA voit le jour le 25 décembre 1945 sous l'impulsion conjointe du général De Gaulle, du ministre des finances René Pelvin et du ministre des Colonies Jacques Soustelle. Les territoires français d'Outre-Mer utilisent déjà le Franc français, avant cette date. Au Cameroun et en Afrique Équatoriale Française (AEF), la Banque de l'Afrique Occidentale (BAO), sous autorisation de la France, y émettait ses monnaies depuis 1920. En 1941, le relais fut pris par la Caisse Centrale de la France Libre (CCFL) puis par la Caisse Centrale de la France d'Outre-Mer (CCFOM) jusqu'au 20 janvier 1955. Sur le plan iconographique, 
les mêmes monnaies émises par la CCFOM seront reprises par l'Institut d'Émission de l'Afrique Équatoriale Française et du Cameroun (IEAEFC) à partir de 1955 avant de s'enrichir des images relatives au vécu quotidien des territoires récepteurs. Ces deux dernières catégories de billets, ainsi que toutes les générations qui suivront pour le compte officiel du Cameroun jusqu'en 2002, ont été retenues comme échantillons pour cette étude. De même, seuls les billets dont l'iconographie présente des scènes de travail ou permet de le déduire, ont été sélectionnés. Le mutisme de l'État dans la signification officielle de ces images ainsi que les difficultés d'accès aux cellules de fabrication de ces monnaies nous ont contrainte à nous limiter une analyse iconographique sémantique des images monétaires.

Les supports monétaires collectés dans des sites de collectionneurs ont été explorés et les images relatives au travail ont été catégorisées ${ }^{7}$. L'analyse iconographique des monnaies s'est faite par la décomposition des images sur les monnaies et l'identification chronologique des thématiques relatives aux femmes et aux hommes en situation de travail. Les faces des monnaies sur lesquelles figurent les images en question ont été sélectionnées et certaines faces ne comportant pas d'images liées au travail ont été maintenues pour compléter l'analyse. Il s'est agi ensuite de faire appel aux techniques d'analyse de l'image de l'histoire de l'art, proposées par Annie Duprat (2007) et Laurent Gervereau (1994) notamment l'identification des contextes interne et externe intrinsèques à l'image. Dans le premier cas, les informations recherchées sont relatives à la date d'émission du billet/pièce (millésime), au nom du graveur/ dessinateur, à l'atelier de fabrication, au commanditaire et au destinataire, à la légende de la monnaie qui tient lieu de nom. Dans le second cas, les thèmes relatifs au travail ont été identifiés et un nom a été donné à chaque image/scène à interpréter. Puis, nous avons décrit l'emplacement (avers, revers, champ, type principal, gauche, droite, centre, haut, bas, etc.) et l'action qui y est figurée. Dans l'interprétation des images, l'histoire nous a permis de situer le contexte régional, national et international ainsi que les enjeux des représentations. De même, des informations numismatiques nous ont permis de nous approprier le glossaire, la signification et les positions graphiques des types d'images. Enfin, la sémiologie de l'image, dans la lignée des travaux de Charles Sanders Peirce sur le signe iconique (Peirce, 1978) et les réflexions sur la lecture sociologique de Roland Barthes (Barthes, 2004), nous a accompagnée dans l'analyse déductive interprétative du corpus. Cette dernière a été possible grâce au recours aux sources secondaires relatives aux messages véhiculés par chaque image. Ces différentes méthodes nous ont donné l'occasion de décrypter et de comprendre ces images. Les échantillons choisis (36 images) sont soumis aux mutations géopolitiques qui seront éclairées au fur et à mesure du texte car, avec la dynamique politique et les enjeux économiques, la structure de l'iconographie monétaire change dans l'espace, impliquant une généralisation ou une localisation du message iconographique. En observant ces monnaies, on peut identifier deux moments forts de ces représentations : la période coloniale française (1945-1960) et la période postcoloniale (1960-2002). 


\section{La période coloniale : genre et expression de l'asservissement des peuples par la métropole entre 1945 et 1960}

7 Après la création officielle du Franc CFA, deux problèmes se posent. Premièrement, les billets émis entre 1945 et 1955 portent la mention Caisse Centrale de la France d'OutreMer. Le Cameroun, étant sous tutelle de la France intègre implicitement cet ensemble géoéconomique de la France. Ces billets sont fabriqués en France et l'iconographie est produite par les artistes français. Le FCFA désigne pendant toutes ces périodes le «Franc des Colonies Françaises d'Afrique ». Les faces et les revers des billets sont communs à tous ces territoires. Deuxièmement, l'iconographie est globalisante, incluant presque tous les territoires et départements Outre-Mer (Antilles, Martinique, Guadeloupe, possessions d'Asie) d'une part, et les possessions d'Afrique Noire d'autre part. Ainsi, le même message circule dans l'empire colonial. Même la couleur semble ne pas catégoriser les possessions françaises. Par conséquent, les considérations que la France a du genre et du travail seraient les mêmes pour toutes ses possessions ou n'auraient simplement pas d'importance.

Images 1. Femme aux seins nus

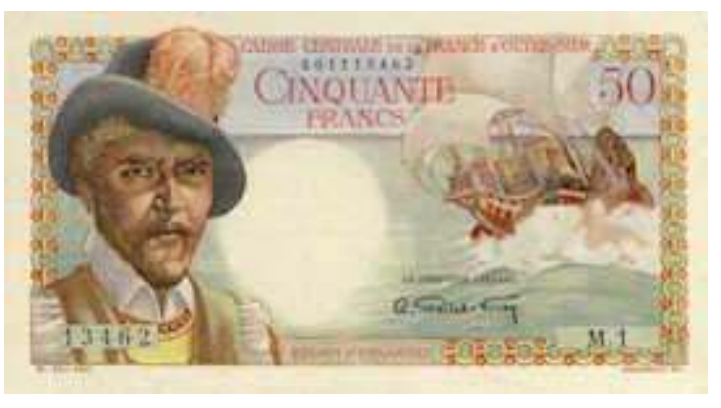

Revers

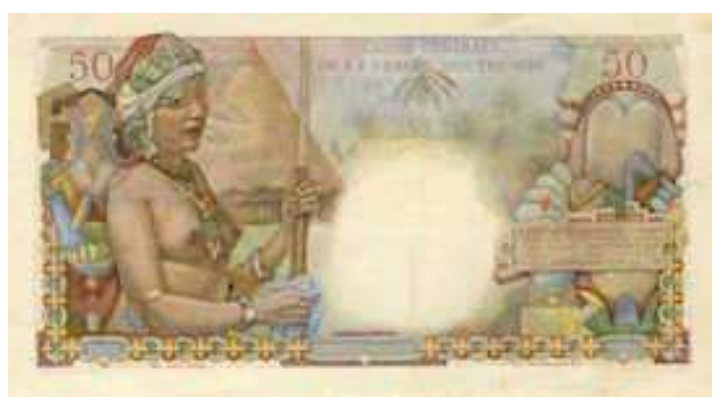

50F, 78x144mm, CCFOM, 1947, revers

(CJLB-COLLECTIONNEUR-

La coupure de 50F, ci-dessus est entourée d'un motif de chaîne sur les deux faces du billet. Elle porte à l'avers le portrait de Pierre Belain d'Esnambuc, explorateur français. Au revers (image 1), figure le buste d'une femme aux seins nus. Seule, elle est près d'un habitat matérialisé par un boucaro, ou case traditionnelle, de forme conique fait de paille ; elle est parée de bijoux, sa main droite nettoie, à l'aide d'un tissu bleu, un bâton 
qui semble surmonté d'une lame en métal. À l'arrière-plan, on aperçoit d'autres cases et des instruments de musique.

Les traits physiques de cette femme, anonyme, sont ceux d'une femme camerounaise telle que représentée dans les affiches coloniales et cartes postales pendant cette période : seins nus, bijoux, bâton, tâches. La carnation pâle, les mamelons rouges et non sombres, puis la façon de tenir le bâton semblent indiquer une identité métissée de cette femme. L'attribution de cette image à l'ensemble des possessions d'Outre-Mer et son anonymat crée des confusions dans la localisation du message mais indique une vision française de la femme générique à toutes les colonies : dénudée, active avec des traits caractéristiques d'une perception occidentale d'une culture traditionnelle africaine indifférenciée suivant la géo-localisation, pourtant suggérée dans les attributs présents sur les billets. En 1947, le port du vêtement était certes vulgarisé, mais dans plusieurs régions africaines et au Cameroun, les femmes dénudées subsistaient encore ${ }^{8}$. Le bâton tenu avec deux mains renverrait au fait de cuisiner, mais il peut aussi renvoyer à la dance, ce qui expliquerait les instruments de musique. Ou encore, la présence d'un épieu pourrait signifier qu'il s'agit d'une chasseresse. Les traits du visage ne semblent guère exprimer le bonheur et la satisfaction. Par sa présence au verso, cette image de femme pourrait être considérée comme un "produit » exotique ou tropical du point de vue du colonisateur à l'avers. L'imprécision circonscrit cette perception du colonisateur à l'ensemble des territoires qui bénéficient de cette monnaie. Enfin, la présence des chaînes symbolise le poids de la puissance coloniale qui n'a pas rompu avec les travaux forcés et tient de main ferme les territoires coloniaux et ses ressources. Cette idée est renforcée par l'image d'un navire et d'un célèbre colonisateur français, gouverneur général des Îles d'Amériques, Belain d'Esnambuc. La femme serait perçue comme une figure d'attraction pour les colonisateurs. Cette construction stéréotypée de l'image de la femme noire sera enrichie par la diversification des portraits de femmes aux seins nus sur d'autres billets de banque et cartes postales?.

Image 2. La femme au sein nu, l'homme et les fruits

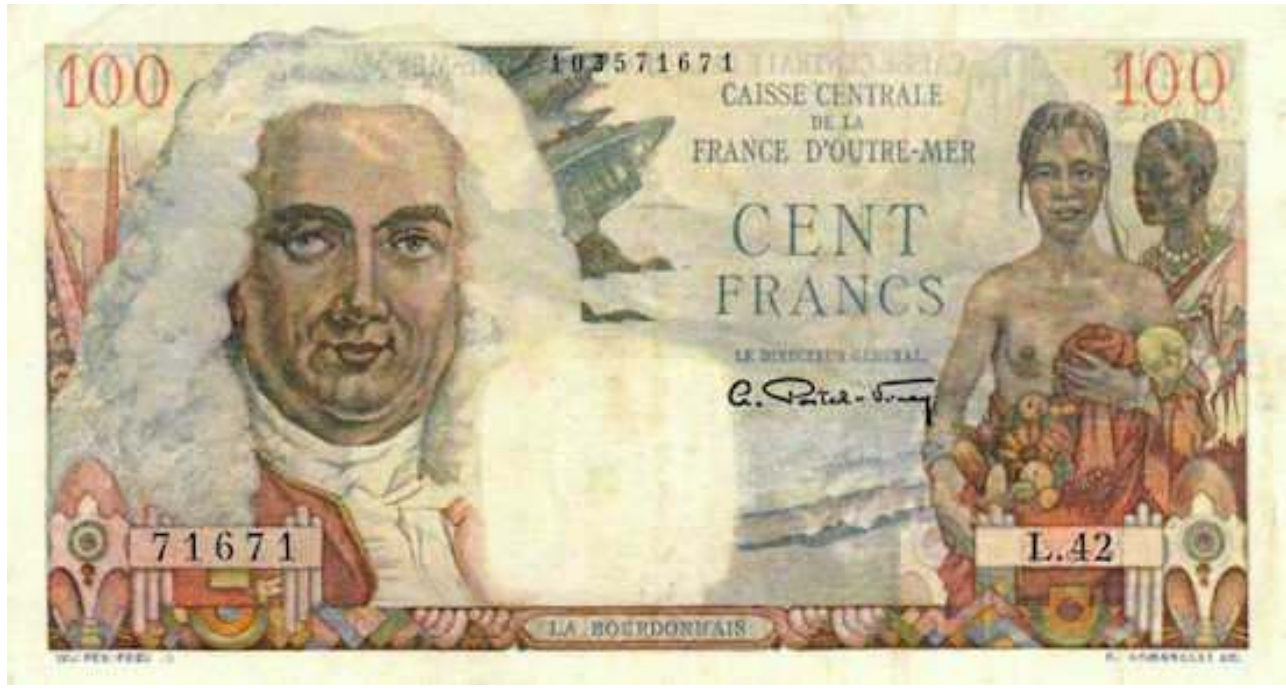

100F, 85x158mm, CCFOM, 1947, avers

(c)JLB-COLLECTIONNEUR- 

marin et administrateur colonial français. À droite, une femme noire au sein nu et dardé, tenant des fruits dans son pagne rouge et cachant son sein gauche avec son pagne grâce à sa main gauche. Derrière elle se tient de profil un homme en tenue traditionnelle.

11 La femme, toujours nue, présente des fruits divers, sans doute issus de l'agriculture et de la cueillette. Dans un premier temps, on peut y lire le rôle nourricier de la femme africaine à travers les aliments qu'elle tient en main et son sein nu qui pourrait rappeler la procréation. Sous un autre angle, il s'agirait d'une image exotique. C'est-àdire qu'elle s'inscrit dans l'ensemble des images construites par les colonisateurs pour afficher les délices des colonies d'une part, et l'état primitif des colonisés d'autre part. Fière, elle fixe l'image tandis que l'homme est de profil. Elle est mise en exergue par rapport à l'homme dont on ne voit qu'une partie du corps. Cela amène à s'interroger sur le regard porté par les colonisateurs sur ces femmes qui, pour la plupart, ont joué le rôle de femmes de ménage tout en étant appréciées par la beauté tropicale de leur corps d'ébène. La femme est représentée dans une situation où elle joue le rôle d'épouse et de femme des champs à travers la cueillette. L'association femme-fruits crée une analogie qui présente la femme comme un produit exotique à consommer ${ }^{10}$. Contrairement à la majorité des traditions africaines qui veut que la femme seconde l'homme, celle-ci est en avant.

Pendant la période coloniale, les femmes sont donc présentées comme le trophée d'une conquête, une ménagère, une chasseresse, un objet de plaisir. L'homme noir est, quant à lui, insignifiant, presque absent. Les femmes sont, par ailleurs, représentées dans les activités domestiques (cuisine, cueillette).

Image 3. Hommes guidant les chariots chargés de cannes à sucre

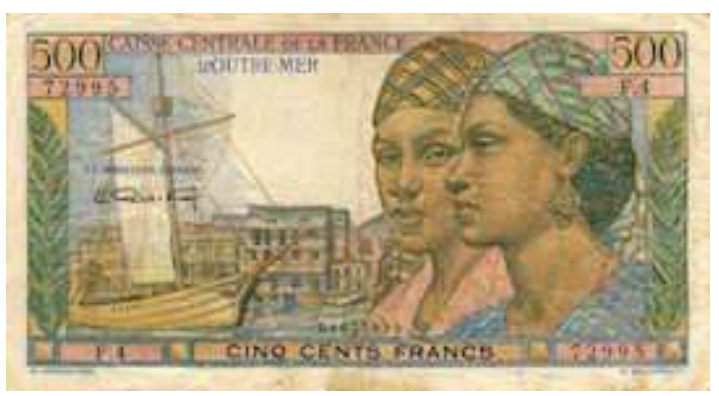

Revers

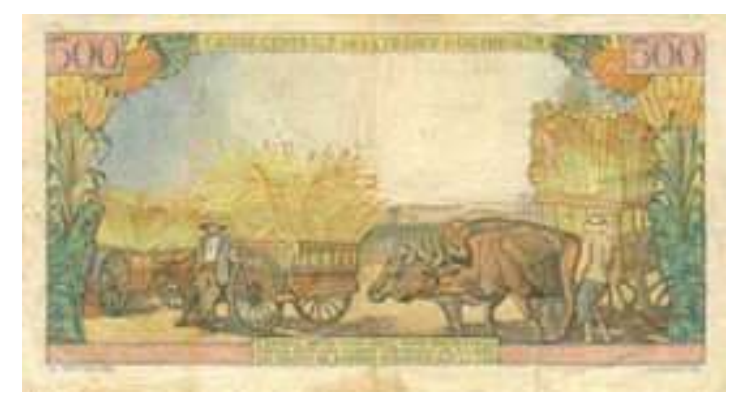

500F, 92x172mm, CCFOM, 1947, avers

(c)JLB-COLLECTIONNEUR- 

chariots de canne à sucre. La canne à sucre fait partie des plus anciennes cultures effectuées par l'homme. Inconnue de l'Europe, sa découverte et sa culture dans les colonies d'Amérique précisément va entraîner une importation de la main d'œuvre d'Afrique pour l'Amérique où les terres étaient propices, ce qui a intensifié l'esclavage. Étant donné les exigences de cette culture (défrichage de grandes superficies à l'aide des machettes, ramassage, transport, nettoyage), les hommes et les femmes y sont fortement impliqués. Mais sur cette image, seuls les hommes sont représentés. Cela s'explique par le fait que ce qui est mis en exergue, c'est la culture de la canne à sucre elle-même et non les acteurs qui la produisent. D'où la petite taille réservée aux hommes dans l'image. Au même moment, le début de l'allègement du travail de l'Homme est mis en exergue par la mécanisation du travail avec des chariots tirés par les bœufs. La représentation de l'effort rentable des hommes s'oppose à l'image des femmes (vêtues) à l'avers.

Image 4. Hommes pagayant sur une pirogue

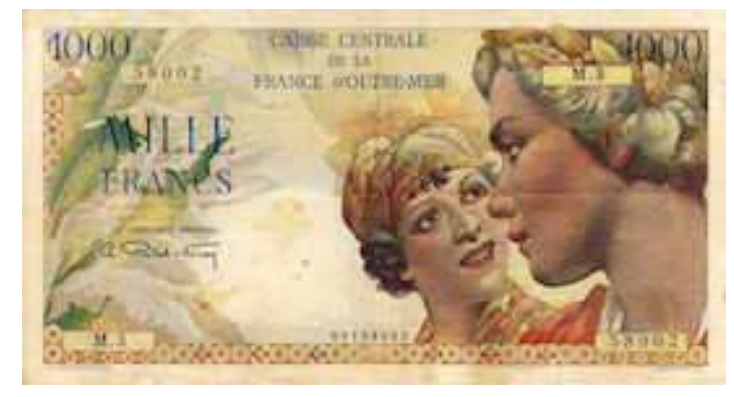

Revers

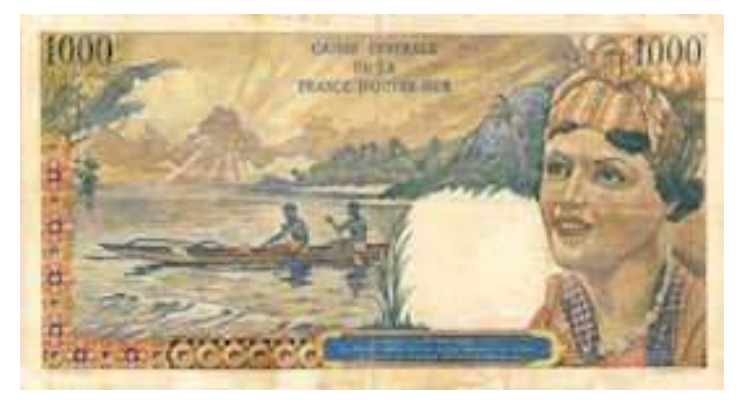

1000F,100x185mm, CCFOM, revers

(C)LB-COLLECTIONNEUR-

En 1947, une coupure de 1000F est émise pour les territoires d'Outre-Mer. Ce sont des représentations de femmes blanches apprêtées et non-identifiées qui composent une partie de la face et du revers. À l'arrière-plan du revers, deux hommes noirs, torses nus, pagaient une pirogue. La pirogue pouvait servir de moyen de transport pour ces dernières et constituait un instrument de pèche très important. Cette image interroge le statut des femmes blanches en colonie. Bien parées, elles semblent préoccupées par les loisirs. 
Image 5. Hommes conduisant une pirogue sur un fleuve

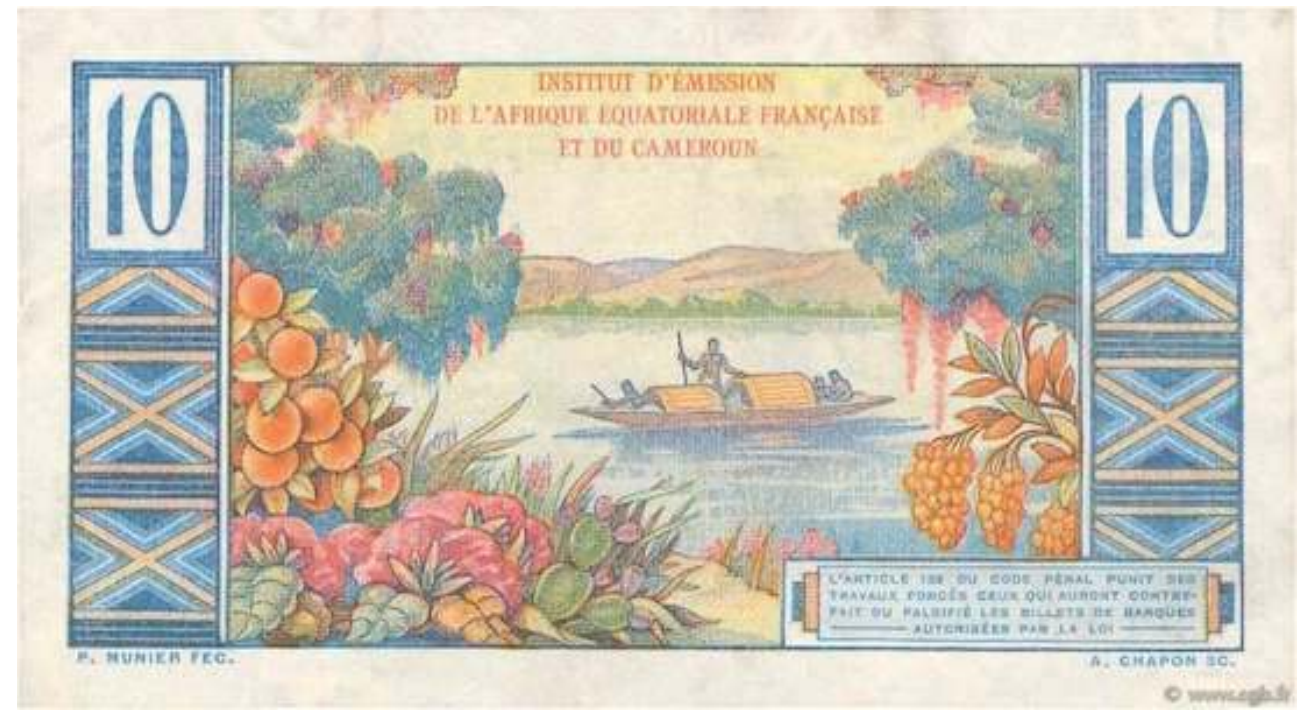

10F, 1957, IEAEFC, 64x118mm, IEAEFC, verso

(C)CGB Numismatique Paris

Cette coupure de Jean-Baptiste Colbert gravée par Hourez et Chapon n'est pas nouvelle dans l'histoire des monnaies coloniales françaises. Elle a été émise par la Caisse Centrale de la France d'Outre-Mer en 1947. Le revers de ce billet présente une jungle et trois hommes dans une pirogue chargée. En Afrique, durant cette période, et particulièrement au Cameroun, les pirogues étaient très utilisées dans les déplacements fluviaux et pour la pêche. Les hommes étaient habilités à la conduire, contrairement aux femmes qui étaient soit des passagères, soit les propriétaires/destinataires des bagages transportés. Cette image montre le quotidien des Africains découverts par les Français dont l'action est matérialisée à l'avers à travers le portrait du mercantiliste et homme politique français Colbert et par la présence des navires à voile.

Image 6. Scène de vie dans un village

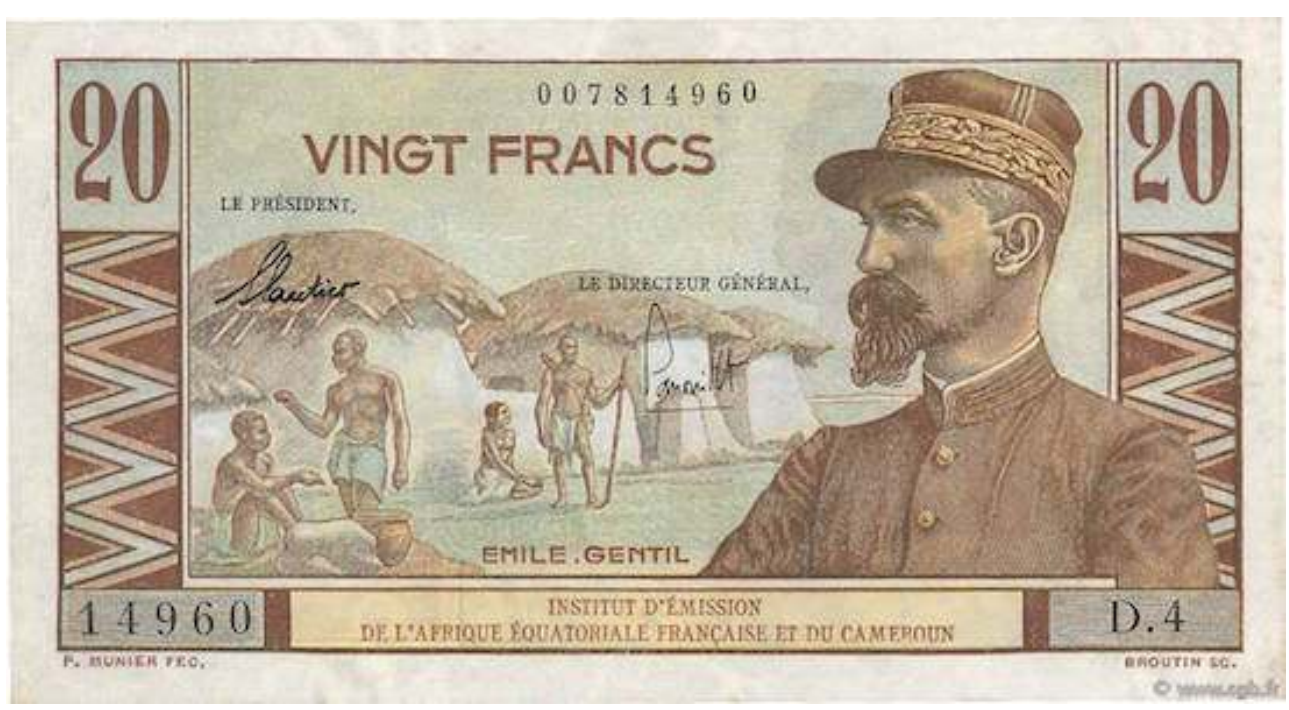

$20 \mathrm{~F}, 70 \times 130 \mathrm{~mm}$, IEAEFC, 1957, avers

(c)CGB Numismatique Paris 


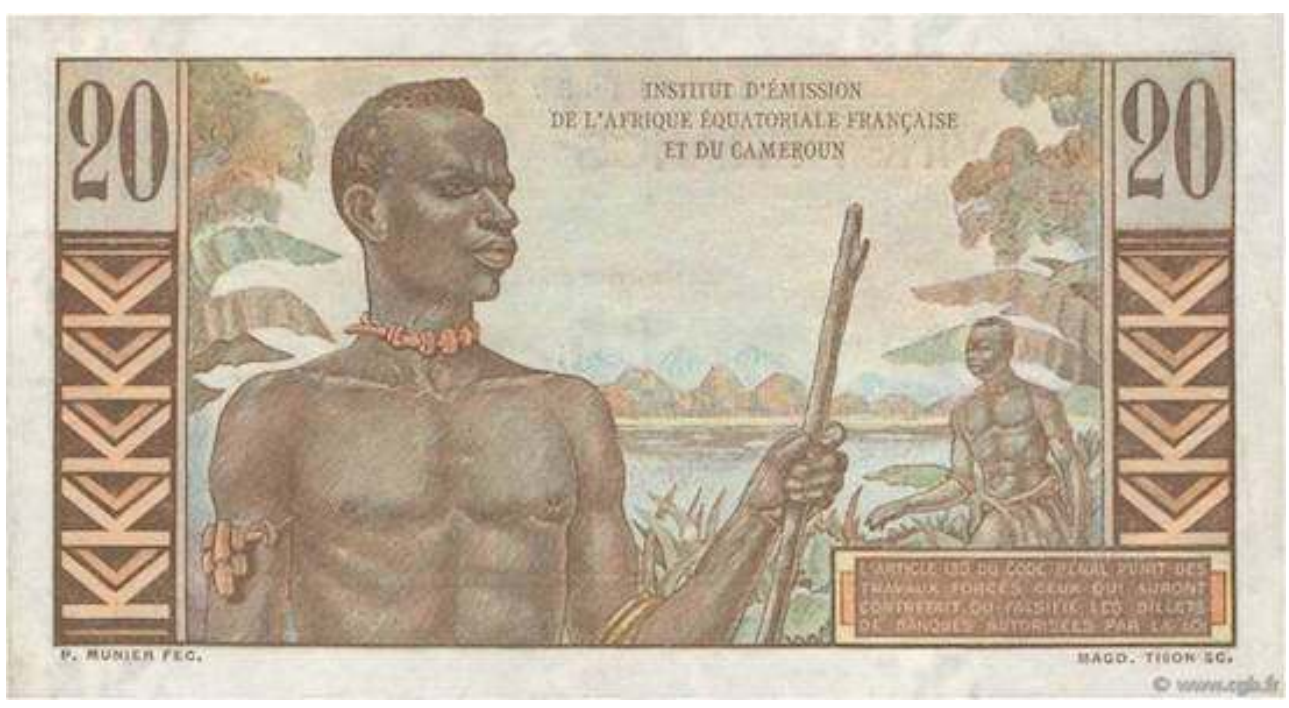

$20 \mathrm{~F}, 70 \times 130 \mathrm{~mm}$, IEAEFC, 1957, revers

(C)CGB Numismatique Paris

L'avers de cette coupure de 20F d'Émile Gentile émise pour la première fois en 1947 (image 6) présente dans son champ deux scènes de vie quotidienne. L'une (scène 2), présente un homme debout avec un bâton, une gourde à boire et son armure pour la chasse. Une femme assise sur le sol, seins nus, vêtue d'un pagne et s'adressant à l'homme, qui, lui, regarde vers l'horizon. Ce mode de communication entre hommes et femmes à l'extérieur de la maison est typique de beaucoup de traditions africaines où la soumission des femmes, sans véritable considération, est capitale (Vincent, 1979). Il en est de même de la répartition des tâches. Les hommes sont appelés à aller chasser le gibier ou cultiver la terre tandis que les femmes sont assignées aux travaux ménagers. Cependant, ce n'est pas propre à toutes les cultures : au Sud- Cameroun par exemple une observation a montré que les femmes sont aussi actives que les hommes dans le domaine de la chasse (Bantous) et dans la production vivrière à travers notamment la constitution des groupes d'initiative commune ou GIC (Ouest-Cameroun) et de parcelles privées (Franquille, 1985).

17 Le revers de ce billet (image 7) présente quant à lui une image particulière : un homme torse nu, portant des fétiches communément appelé « grigri » et des bracelets au coude gauche. Il tient par cette main un bâton. Le regard profond et inquiet, il est suivi par un autre près d'un fleuve. Sa parure l'identifie à un chasseur. Sa tenue "primitive" renforce l'idée que le colonisateur a du camerounais et de l'AEF. La camerounaise en est absente. Pourtant, les femmes jouent un grand rôle dans l'exploitation forestière : chasse, bois, cueillettes etc. Un rapport sur le genre dans le déboisement et la dégradation des forêts au Cameroun (REFACOF, 2014) le démontre :

«Au sein de la société traditionnelle Bantou et pygmée, la femme est la véritable cheville ouvrière au regard de ses longues journées de travail et de la diversification de ses activités. En effet, elle est fortement impliquée dans l'utilisation des ressources forestière à travers leur exploitation, transformation, autoconsommation et commercialisation. "

18 Ces représentations qui circulent sur le territoire camerounais n'épousent pas les réalités spécifiques des différents peuples qui y cohabitent. Certains fonds communs 
existent dans les types de tâches accordées à chaque sexe mais dans la réalité les implications des uns et des autres vont au-delà : agriculture, élevage. De plus, en 1957, une forte répression s'exerce au Cameroun contre les mouvements nationalistes. Les femmes tout comme les hommes participent activement à cette guerre ${ }^{11}$. C'est en 1958, que les images monétaires mises en circulation par l'IEAEFC présentent des images plus locales, par rapport à l'empire toute entier.

Image 8. Femme cueillant du café

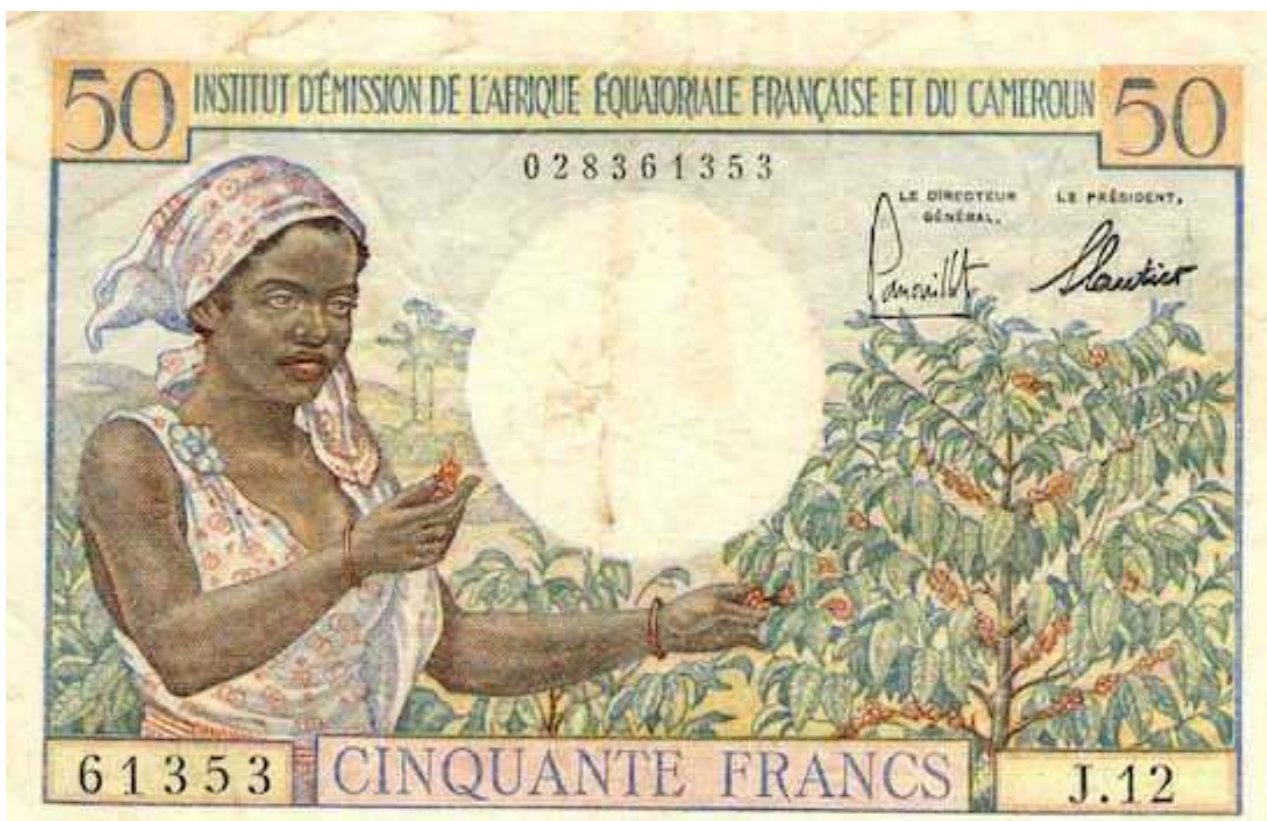

50 Francs, 76x117mm, IEAEFC, 1958, avers

(c)Multicollect.net 


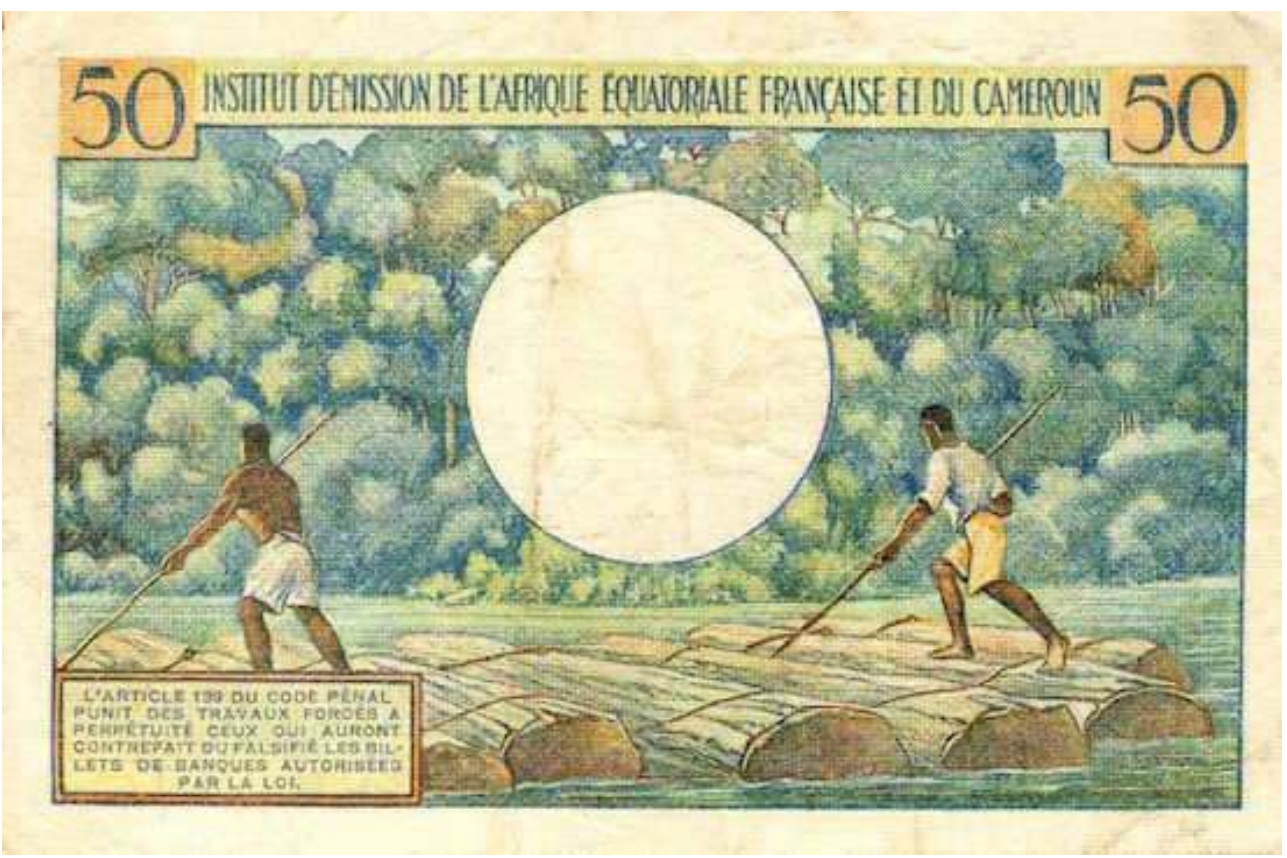

50F, 1957, IEAEFC, 76x117mm, revers

(C)Multicollect.net porte deux bracelets en tringle de laiton (monnaie précoloniale à l'Ouest-Cameroun). Le café est l'une des matières premières cultivées au Cameroun et symbolise, pendant la colonisation, une agriculture majoritairement vouée à l'exportation. La culture de cette matière première était une affaire d'homme ainsi que l'entretien de ses champs et la commercialisation (Guétat-Bernard, 2011), tandis que les femmes étaient impliquées dans les récoltes et le tri. Cette répartition culturelle des tâches va s'estomper avec la valorisation des cultures vivrières et l'implication croissante des femmes dans l'agriculture (Guétat-Bernard, 2011). Par ailleurs, c'est la première fois qu'une femme apparait dans une activité économique rentable sur la monnaie coloniale française en circulation au Cameroun. Cela peut s'expliquer par la conjoncture politique de cette période caractérisée par les mouvements indépendantistes et le besoin de poursuivre l'agriculture de traite dans les possessions françaises. L'attention du lecteur est détournée de son seul physique et centrée sur son travail. La femme n'est plus exposée, mais perçue comme une productrice de biens.

L'exploitation forestière mise en exergue dans la deuxième image (image 9) semble une activité essentiellement masculine, tant elle nécessite de la force habituellement/ culturellement attribuée aux hommes. Avant l'indépendance, l'exploitation forestière était destinée en majorité à l'exportation car le Cameroun ne disposait pas d'usine de transformation. Les Français vont donc entreprendre une exploitation profonde du bois au Cameroun, comme en RCA et au Gabon. La taille des grumes et les dangers que 
représente leur coupe, amène à comprendre l'absence des femmes dans les représentations genrées de cette activité. Néanmoins, l'exploitation du bois ne s'arrête pas aux grumes qui sont exportées. Le transport du bois se fait aussi sur la tête ou sur le dos. L'exploitation du bois de chauffe, pour l'artisanat ou pour la construction concerne beaucoup de femmes (REFACOF, 2014).

Ces deux représentations renforcent, en effet, l'image qui s'est développée autour du travail en Afrique, à savoir que l'agriculture est un univers féminin, tandis que la forêt est un univers masculin (Barbier, 1985). Pourtant, chaque sexe a un rôle dans chaque domaine selon son intérêt et ses obligations. Les produits d'exportation sont d'ailleurs davantage mis en exergue étant donné que depuis la crise de 1929, la France s'est totalement repliée sur ses colonies pour s'approvisionner en matières premières de consommation et d'industrie (Gérardin, 1989). Les hommes et les femmes sortent de leur culture concernant l'ordre des travaux pour se mettre chacun selon sa force au service de la métropole.

Images 10. Femme sahélienne

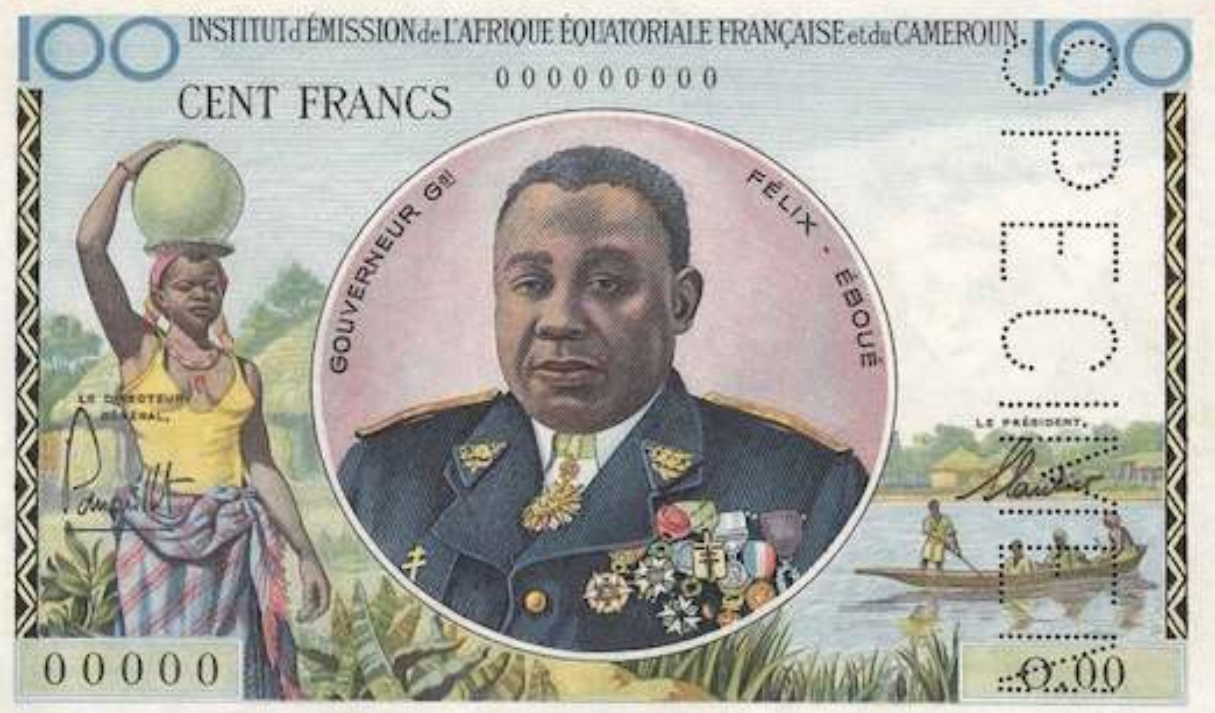

100F, 83x 128mm, IEAEFC, 1957 et 1961, avers

(C)GB Numismatique Paris 
Image 11. Homme conduisant les passagers dans une pirogue

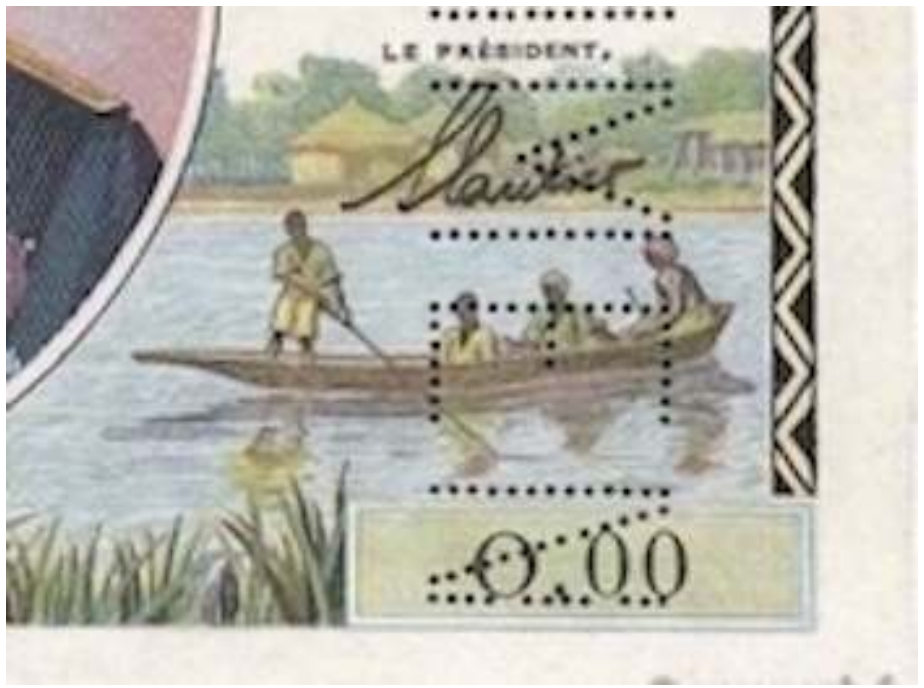

100F, 83x 128mm, IEAEFC,1957 et 1961, avers

(c) GB Numismatique Paris.

Image 12. Hommes en activités dans un port

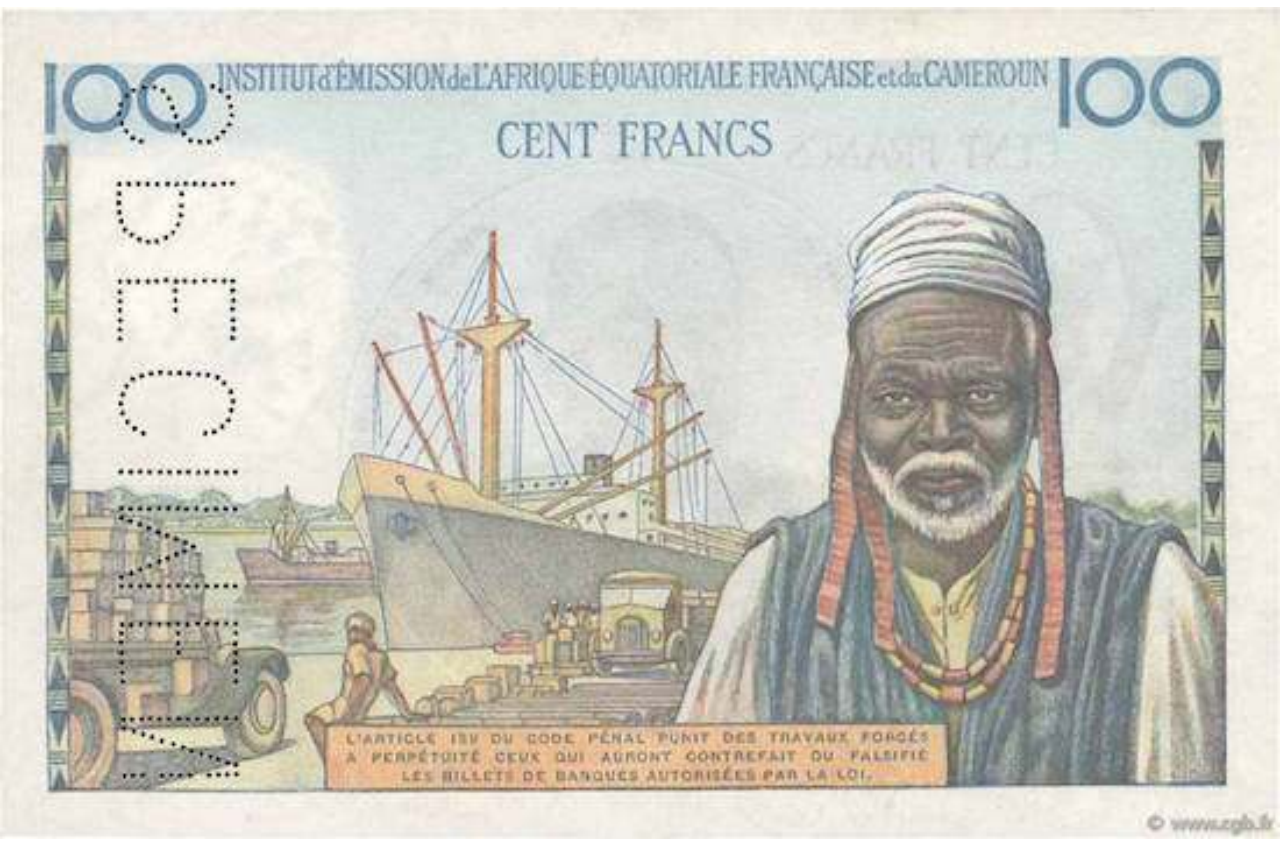

100F, 83x 128mm, IEAEFC,1957 et 1961, revers

(C) CGB Numismatique Paris

La coupure de 100F ci-dessus porte l'effigie Félix Eboué, gouverneur général de l'AEF et administrateur colonial français. Dans le champ de l'avers (image 10), à gauche, une jeune femme, en pagne traditionnel enroulé sur ses hanches porte un haut décolleté exposant une partie de ses seins. Elle a sur la tête un canari soutenu par sa main droite et posé sur un plat tissé traditionnellement. Il s'agit d'un ustensile de cuisine pour préparer les repas, conserver de l'eau ou autres produits. Il existe, en effet, un lien très étroit entre ces travaux domestiques : pour cuisiner, il faut par exemple aller chercher de l'eau ou aller écraser les céréales quand elles ne sont pas pilées. Derrière elle, un 
village sahélien avec des cases de paille. L'image 11, quant à elle, fait référence à un homme pagayant une pirogue dans laquelle sont assis un homme et deux femmes. La pirogue est, généralement, pagayée par des hommes, mais au Sud-Cameroun et à l'Extrême Nord, les femmes pagaient aussi, seules ou avec d'autres femmes, des pirogues transportant des personnes ou des biens. Par ailleurs, signe de l'extension de la France au Nord-Cameroun, des images du Sahel apparaissent pour la première fois sur cette série de billets.

Sur le revers, l'image d'un port est mise en exergue (image 12). Des camions chargés, transportent vers le bateau stationné, des produits d'exportation. Au sol, des grumes attendent d'être chargées par les hommes qui sont situés à côté. La présence d'un vieillard symbolise la durée des rapports économiques et sociaux avec les peuples concernés. Les femmes sont absentes de cette scène de portage et de chargement de bateaux.

Image 13. Femme dans une cacaoyère

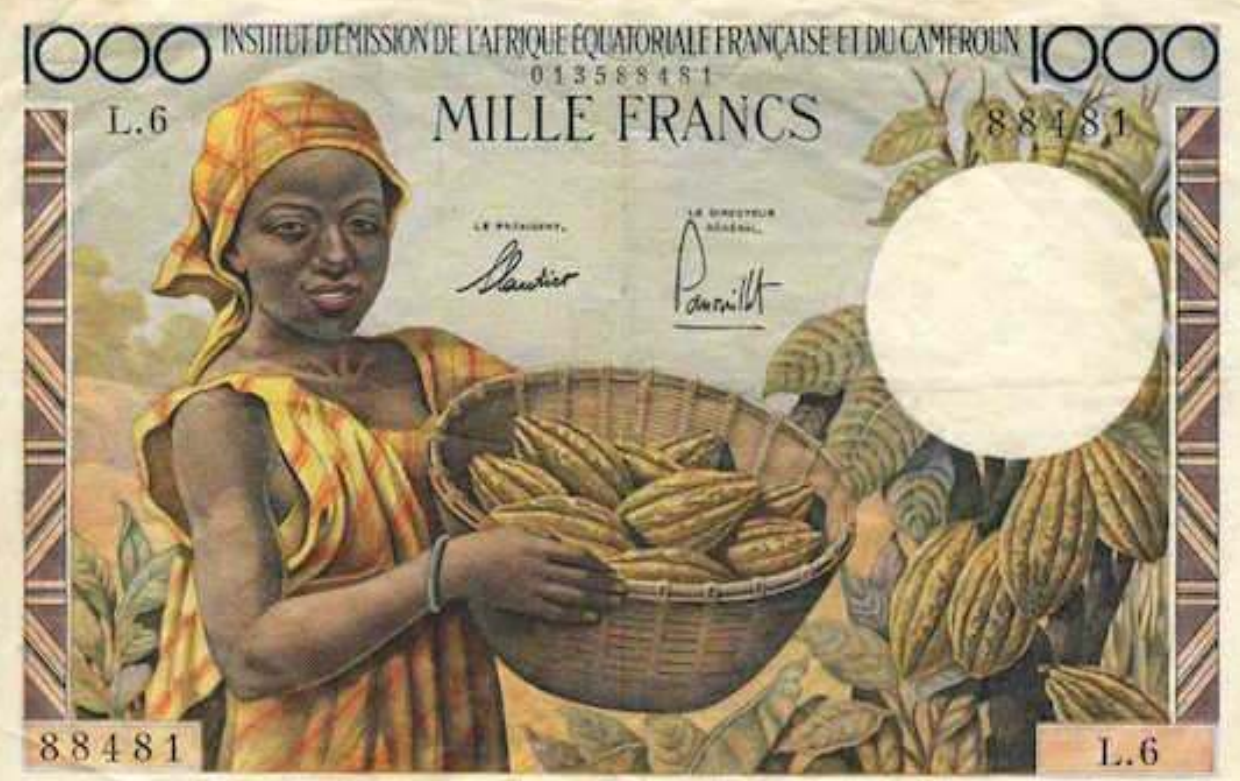

1000F, 103x159mm, 1958, IEAEFC, avers

(c)Multicollect.net 


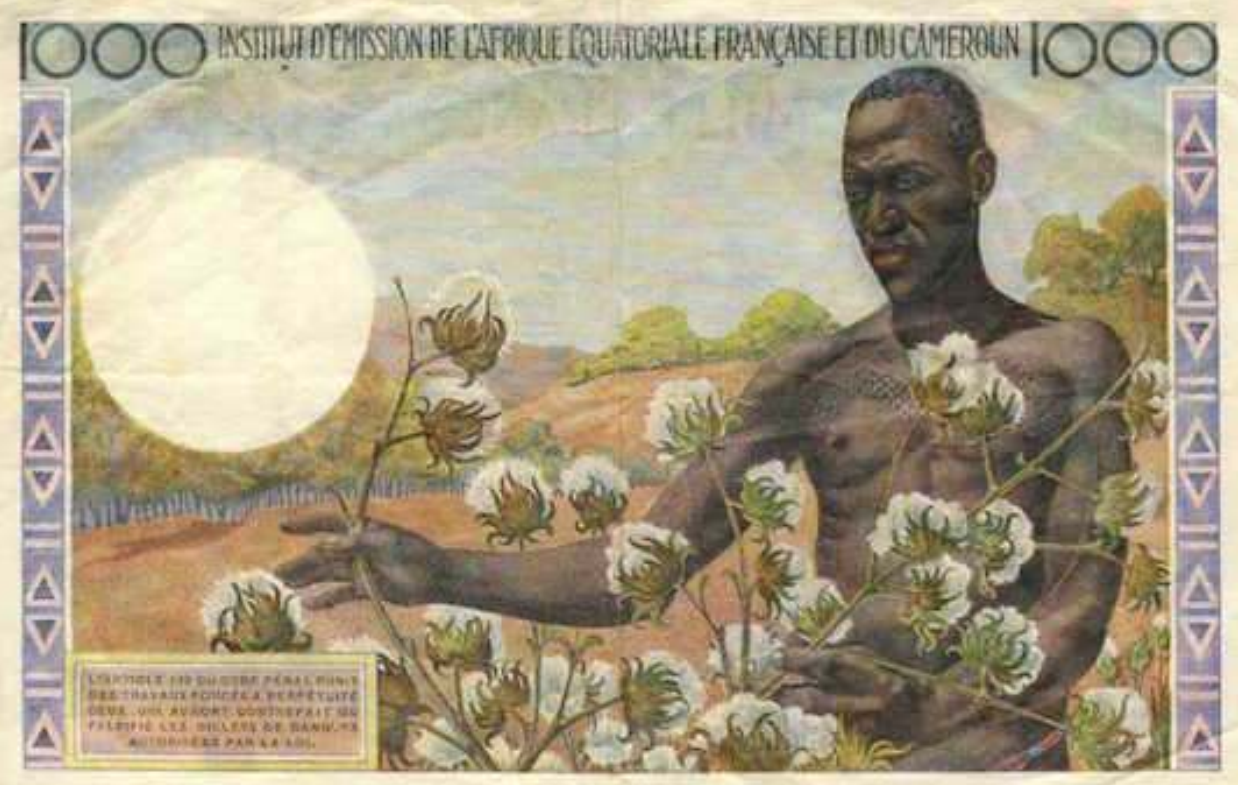

1000F, 103x159mm, 1958, IEAEFC, revers

(C)Multicollect.net

L'avers du billet de 1000F ci-dessus a pour type principal une jeune femme souriante tenant un panier de cacao (image 13). Elle est vêtue d'un kaba, une tenue traditionnelle commune aux femmes du littoral, du Sud, de l'Ouest et de l'Est-Cameroun. Sur le revers, un homme, torse nu, récoltant du coton, matière première cultivée au Nord-Cameroun.

Il s'agit donc d'une femme du Sud-Cameroun. Elle est en pleine activité dans une plantation de cacao. Le message des images met en valeur le rôle des femmes dans l'économie coloniale et semble promouvoir le travail féminin. Le cacao est aussi l'une des matières premières stratégiques cultivées au Cameroun en 1958. De même, l'homme est représenté dans son labeur à travers la récolte du coton qui s'effectue sous le soleil et torse nu (image 14). L'homme au nord et la femme au sud sont reconnus comme étant les principaux acteurs de l'économie coloniale. Cependant, on peut relever une permutation de la nudité : alors que la femme est de plus en plus présentée habillée, c'est l'homme qui est dévêtu : le torse nu renvoyant à sa force physique.

Pendant cette première période, on note tout d'abord la présence des hommes et des femmes des deux couleurs. Les femmes noires sont les premières à apparaître et sont plutôt affectées aux tâches ménagères. Avec le temps, elles sont intégrées dans les tâches commerciales pour la colonie. Les hommes en situation de travail apparaissent après les femmes. D'abord effacés, ils prennent de l'importance dans l'iconographie monétaire car ce sont eux qui effectuent les tâches d'exportation les plus importantes, notamment le transport des produits par voie fluviale ou maritime.

Le produit du travail présenté dans ces images ne « profite " pas aux peuples colonisés mais essentiellement à la métropole. Il s'agit en majorité des activités liées à la prospérité de cette dernière, tandis que les autres activités quotidiennes des peuples illustrés sont déduites. Aucune illustration ne permet de lire clairement les tâches traditionnelles ou culturelles de ces peuples. Leur identité est confondue à leur apport dans l'économie coloniale. De même, la construction du genre qui est faite ne 
correspond ni à la conjoncture occidentale sur la question, ni, encore moins, à la réalité historique de ces peuples décrite partiellement. C'est donc dire que ces billets sont une démonstration de force des colonisateurs qui y apparaissent à travers des figures emblématiques. L'avènement de l'indépendance va entraîner une métamorphose du champ du travail en fonction des nouvelles attentes de développement.

\section{La période postcoloniale : entre valorisation et exclusion de la femme du champ professionnel}

\subsection{Les billets de la BCEAC (1960-1972) : masculinisation du travail et exclusion de la femme dans l'économie}

Avec l'indépendance proclamée au Cameroun le 1er Janvier 1960, les billets émis par la Banque Centrale des États de l'Afrique Équatoriale et du Cameroun ont la particularité de porter une face individuelle à chaque État et un revers commun. Le Cameroun a donc des images personnalisées sur la face de ces billets. Le Franc CFA désigne dès 1958 Franc de la Communauté Financière d'Afrique.

Image 15. Quotidien dans un village

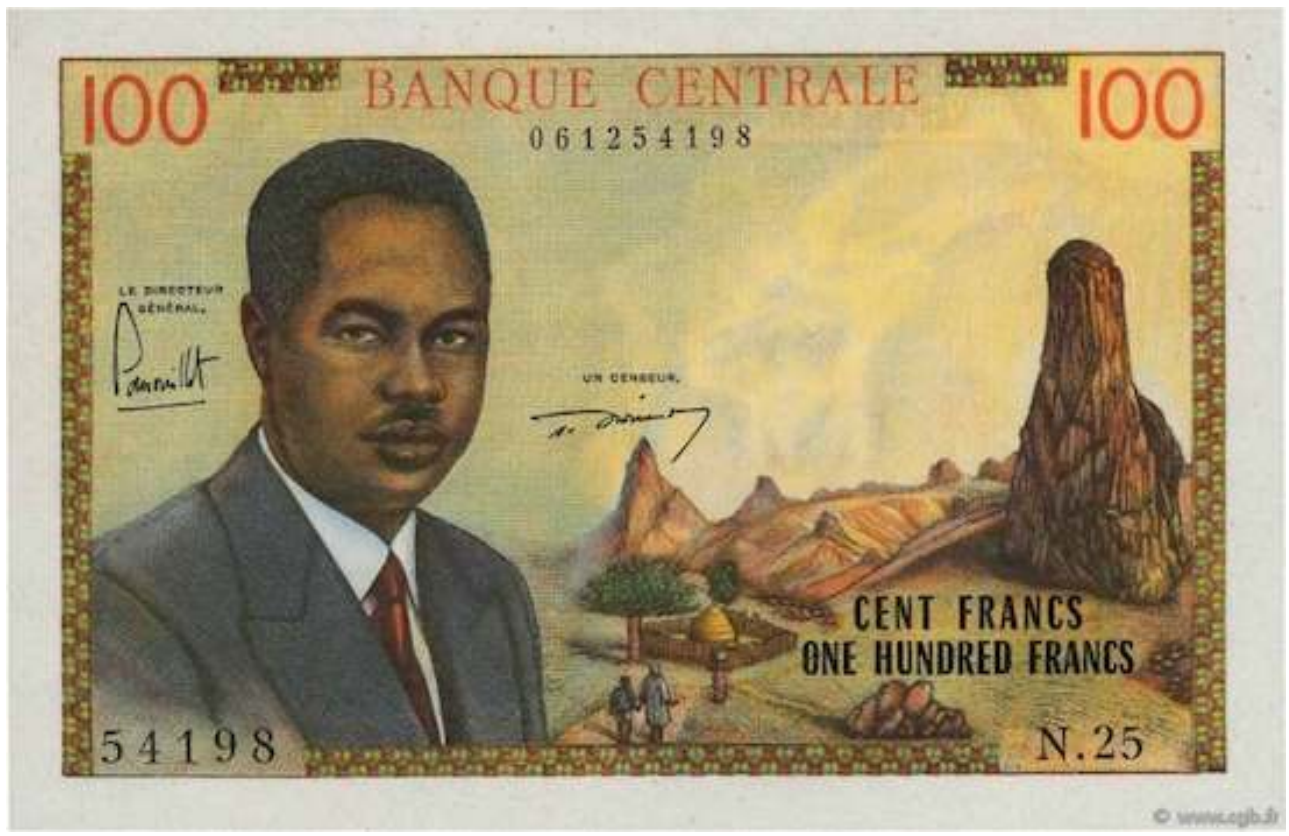

100F, 83x128mm, 1963, BCEAEFC, avers

(C)CGB Numismatique Paris 


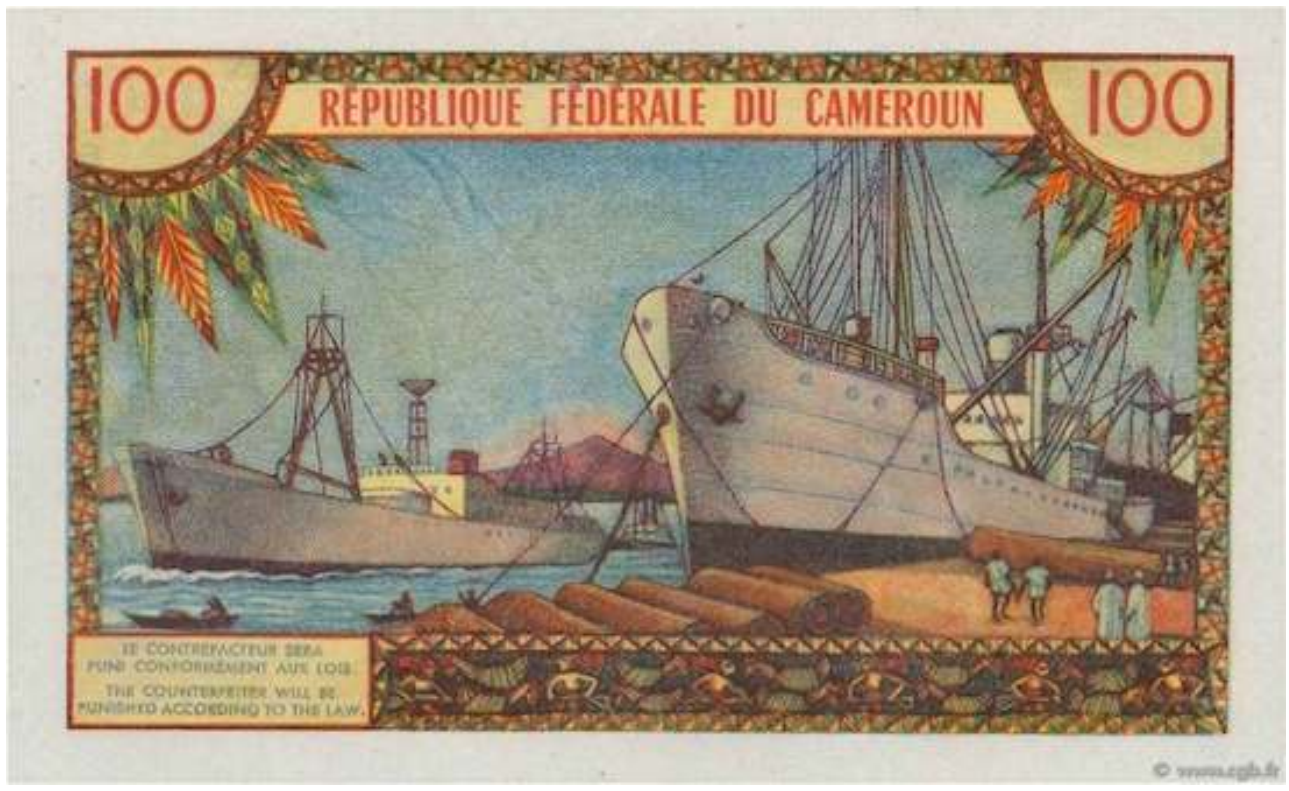

100F, 83x128mm, 1963, BCEAEFC, revers

(C)CGB Numismatique Paris

Après l'indépendance du Cameroun, le billet de $100 \mathrm{~F}$ ci-dessus est émis avec une iconographie personnalisée (image 15). Elle montre un village sahélien où un homme et un jeune écolier avancent vers le village main dans la main, dans une imagerie renvoyant à la causerie, tandis qu'une femme en sort avec un récipient sur la tête. Dans cette partie du Cameroun, les femmes dans leurs activités culinaires sont toujours à la recherche de l'eau. L'homme est chargé d'assurer le reste de provisions. Cette scène décrit un paysage familier au président Ahmadou Ahidjo dont le portrait figure au premier plan de l'image : ce qui serait un hommage à sa région d'origine, le grand Nord $\mathrm{du}$ Cameroun. Sur le revers (image 16), une scène d'exportation du bois est une fois de plus illustrée et ce sont des hommes en gandoura/boubou qui y sont représentés. En opposition au rôle de la femme sur l'avers, qui est assigné à la cuisine, l'homme va à l'école, voyage et travaille dans les ports. 


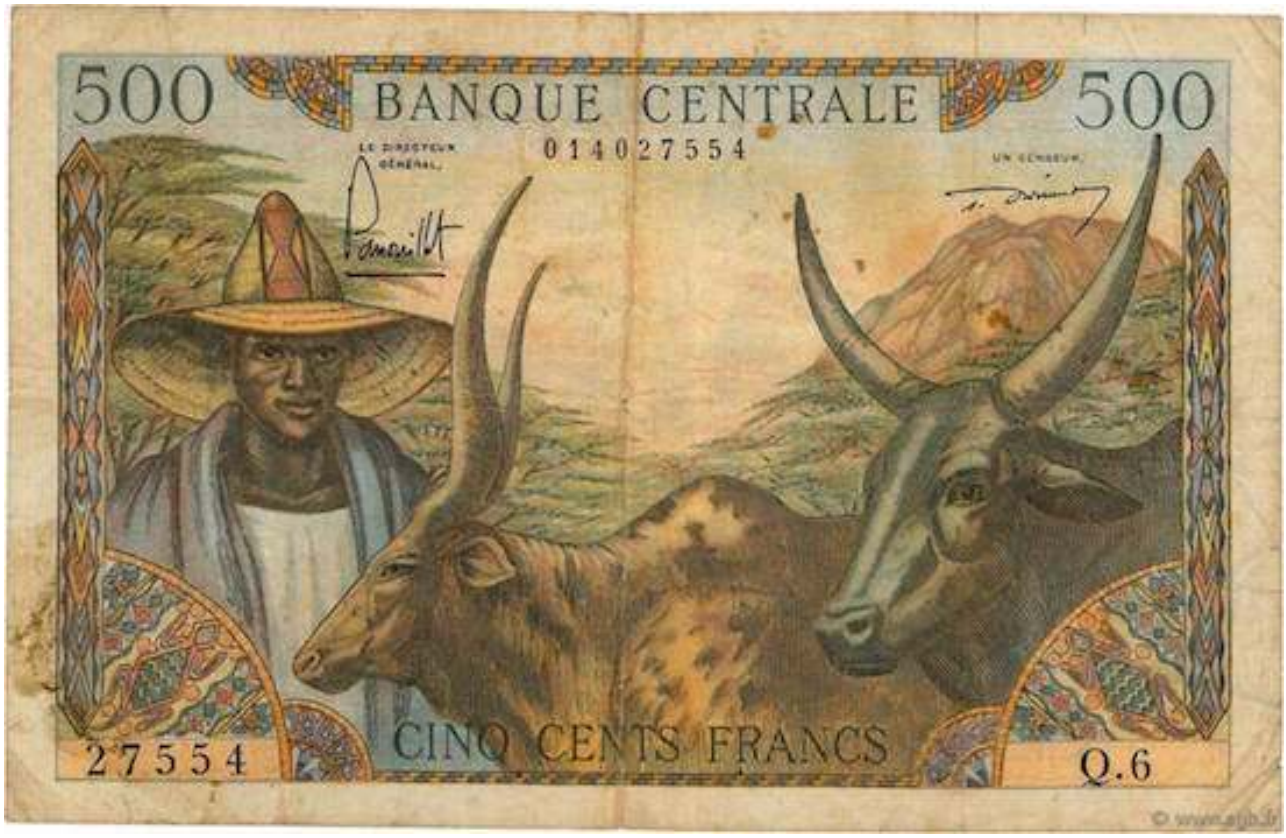

500F, 90x139mm, 1962, BCEAEC, avers

(c) GB Numismatique Paris

Image 18. Transport de bananes/plantains par des hommes dans une bananeraie

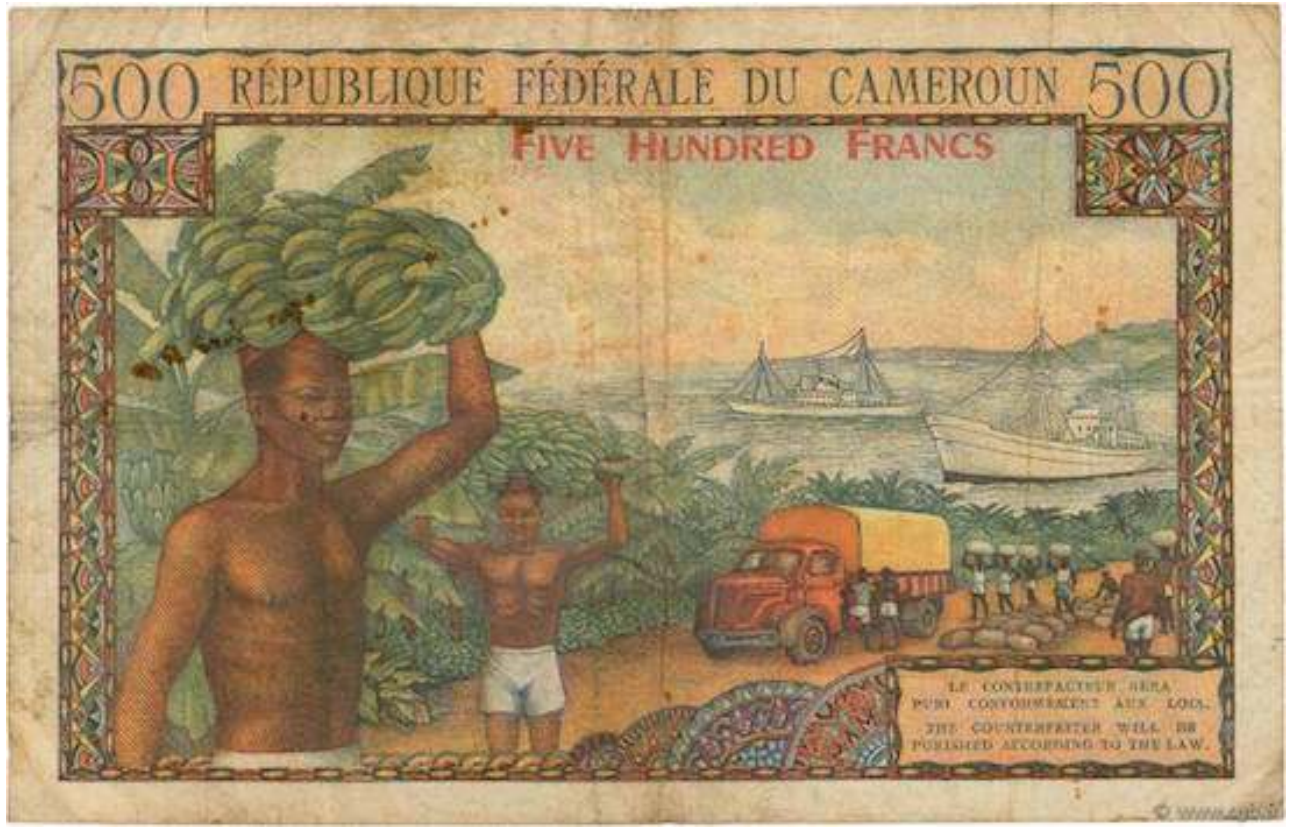

500F, 90x139mm, 1962, BCEAEC, revers

(C)CGB Numismatique Paris

La face de cette coupure de 500F présente en premier plan deux taureaux aux cornes imposantes et un berger les fixant (image 17). Dans le champ du billet, un paysage montagneux avec les arbres de type sahélien. Souvent négligé et mal payé, le berger exécute un travail de longue haleine en conduisant les troupeaux à longueur de journée dans des pâturages; il les protège des bêtes sauvages, des accidents et les soigne. Ce 
travail a toujours été masculin, à quelques exceptions près, particulièrement chez les peulh du Nord-Cameroun. Le berger travaille à plein temps et parcourt de grandes distances où il affronte les voleurs et des bêtes sauvages (Boutrais, 1994 ; 1999).

Le revers de la coupure de 500F ci-dessus émis en 1962 (image 18) montre un homme portant un régime de plantain sur la tête, suivi d'un autre. Dans le champ du billet, on observe une voiture en plein chargement et des hommes en file indienne chargés chacun d'un régime sur la tête. La présence des flottes près de la bananeraie montre qu'il s'agit d'une zone côtière et rappelle l'exportation de la banane-plantain. C'est une fois de plus une tâche exclusivement masculine qui est donnée à voir. La récolte d'une bananeraie s'enchaîne à la hâte pour éviter la dégradation rapide du produit. Les charges à transporter sont réputées trop lourdes pour être assurées par des femmes.

Image 19. Homme transportant un panier de cacao

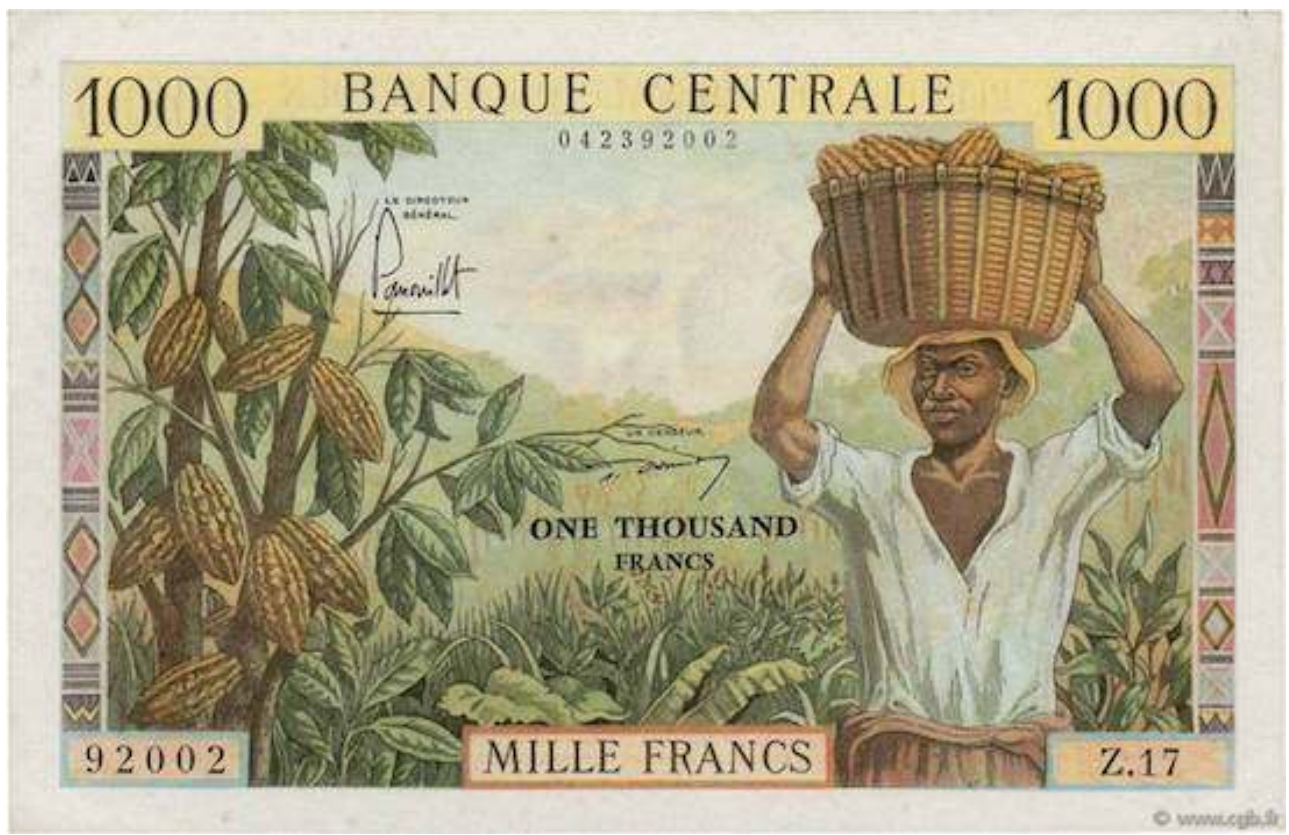

1000F, 103x159mm, 1962, BCEAEC, avers

(C)CGB Numismatique Paris 


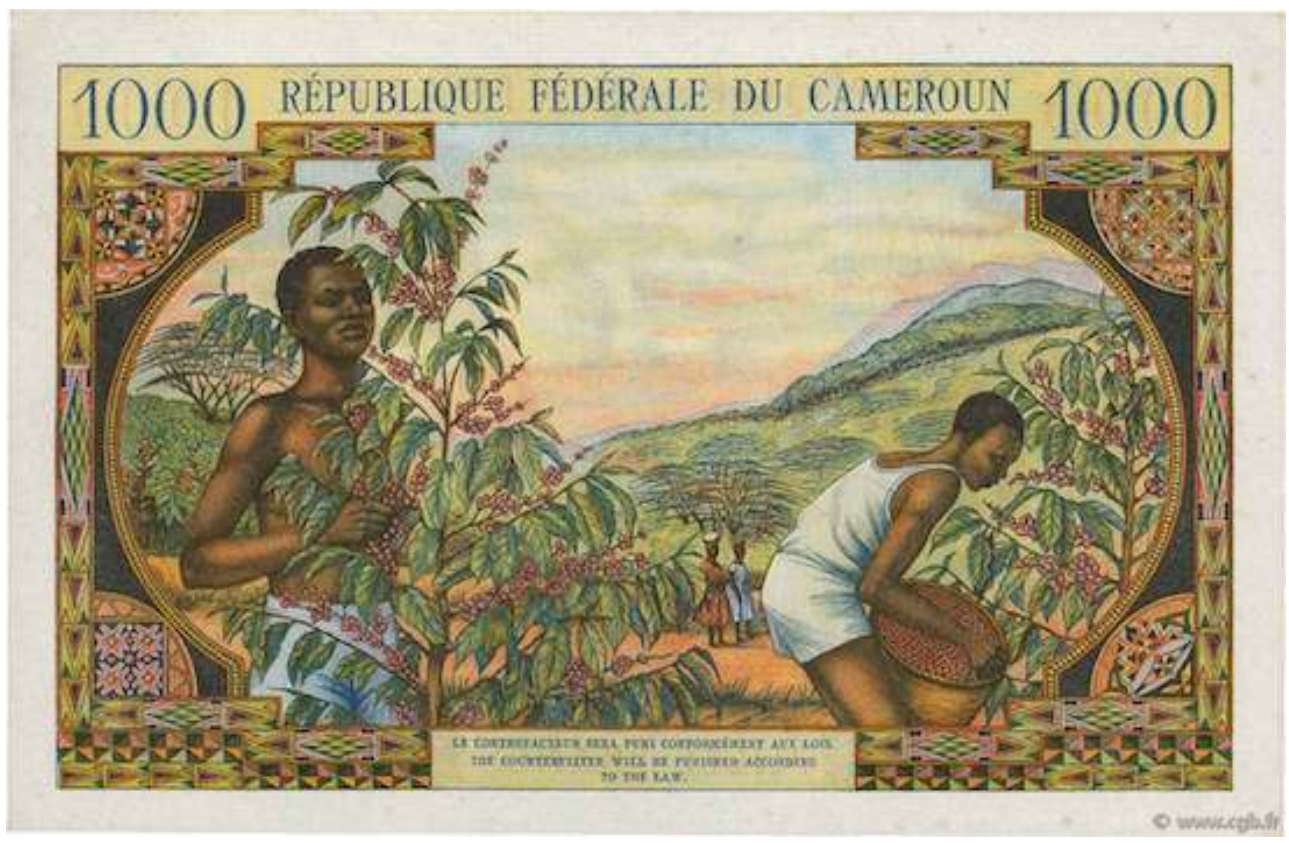

1000F, 103x159mm, 1962, BCEAEC, revers

(C)CGB Numismatique Paris

La coupure de 1000F ci-dessus exclusivement dédiée au travail, présente à l'avers une cacaoyère et un homme portant un panier rempli de cabosses de cacao (image 19). Audelà de la récolte, le transport est fondamental dans le processus de production et de commercialisation de cette matière première. La force musculaire, plus volontiers attribuée aux hommes qu'aux femmes, s'avère nécessaire. Le revers de ce billet montre aussi deux hommes en pleine récolte de café (image 20). Au fond de l'image, on aperçoit deux femmes en marche qui semblent discuter. Ici c'est le travail de l'homme qui est valorisé.

Après l'indépendance, les femmes en situation de travail sont quasi absentes et lorsqu'elles apparaissent, elles sont en arrière-plan. L'homme est au centre de l'action dans l'agriculture, l'élevage et l'exportation. D'une part, cette situation pourrait s'expliquer par l'appartenance religieuse et culturelle du chef de la Nation qui assigne les femmes à rester sous l'ombre de leur époux et hors de la sphère publique. Néanmoins, c'est toujours la France qui décide du choix et des réalisations de ces images ${ }^{12}$. L'ensemble de l'iconographie de cette période met l'accent sur l'image du nouveau chef d'État qui doit être accepté par le peuple, ainsi que son idéologie fondée sur l'« unité nationale ». D'autre part, le travail est très présent dans l'iconographie. Il renvoie aux efforts de développement à travers les secteurs clés de la vie économiques qui sont à cette période l'agriculture (cacao, café, plantain), le bois (grumes) et l'élevage (bœufs). Ces secteurs sont identiques à ceux qui ont nourri la période coloniale. Les acteurs sont les mêmes mais les femmes y apparaissent de plus en plus uniquement comme des figurantes. La représentation de leur travail sur les monnaies officielles du Cameroun semble ne plus intéresser autant qu'auparavant. . Pourtant, les billets d'autres pays de l'AEF montreront l'action des femmes dans le commerce. 


\subsection{Les billets de la BEAC et la structuration du travail : les métiers d'homme et les métiers de femme}

En 1972, le Cameroun et les pays de l'AEF s'unissent au sein de la CEMAC et le bureau d'émission est ramené en Afrique, avec notamment la création de la Banque des États de l'Afrique Centrale. Cependant, les monnaies sont toujours fabriquées en France; l'iconographie est réalisée par les français et mise en circulation par la BEAC. Le Franc CFA désigne pour ces pays le Franc de la Coopération Financière en Afrique Centrale.

Image 21. Homme conduisant un engin agricole

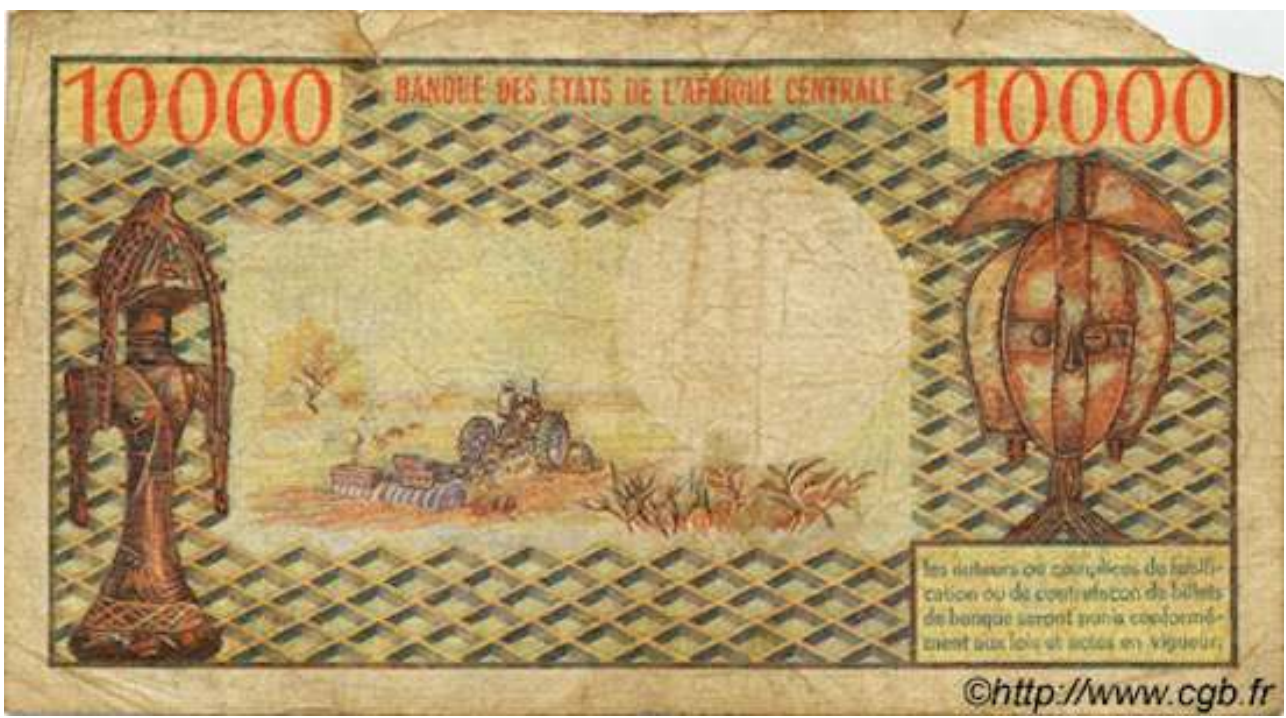

10000F, 91x169mm, 1971, BCEAEC, revers

(c) GB Numismatique Paris

La coupure ci-dessus est le revers du billet de 10000 Francs dont l'avers porte l'effigie du président Ahmadou Ahidjo ${ }^{13}$. Le revers, quant à lui, évoque l'évolution des techniques agricoles avec la présence d'un tracteur en activité dans un champ. Cet engin est conduit par un homme et le paysage, semblable à la savane, ferait référence au nord, voire l'extrême-nord du Cameroun. 


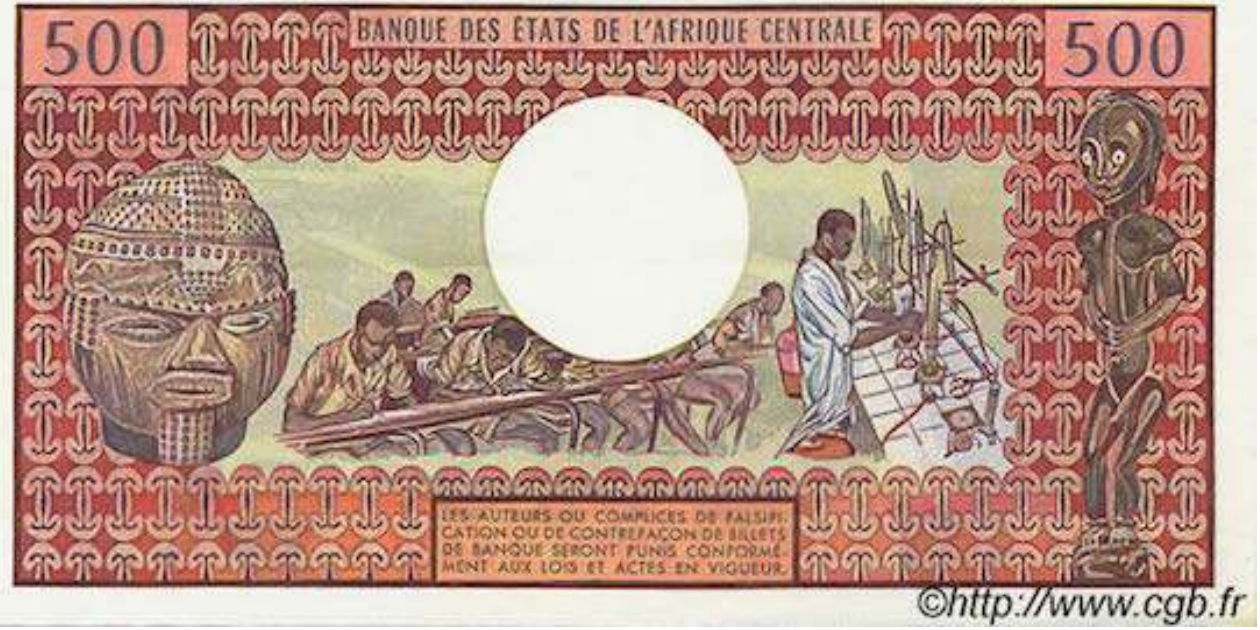

500F, 81x157mm, type 1974, BEAC, revers

(c) CGB Numismatique Paris

En 1974, le revers de la coupure de 500 F - République unie du Cameroun - présente une classe où les élèves, assis sur des tables-bancs, suivent le professeur en train de réaliser des expériences de laboratoire. Il s'agit essentiellement d'hommes mais c'est une autre image du pays qui est ici représentée en mettant en scène une modernité qui rompt avec les représentations précédentes, perçues comme plus traditionnelles. En fait, en 1974, le taux de scolarisation des femmes est presque nul à cause de facteurs socioculturels comme par la prééminence accordée aux hommes dans l'éducation et la formation et la pauvreté. L'éducation des filles n'est quasiment assurée que dans la cellule familiale par leur mère (Fonkoua, 2006). Leur destin le plus probable est le mariage, précoce dans certaines régions (Kembe Milolo, 1986).

Image 23. Activités dans la commercialisation des produits vivriers

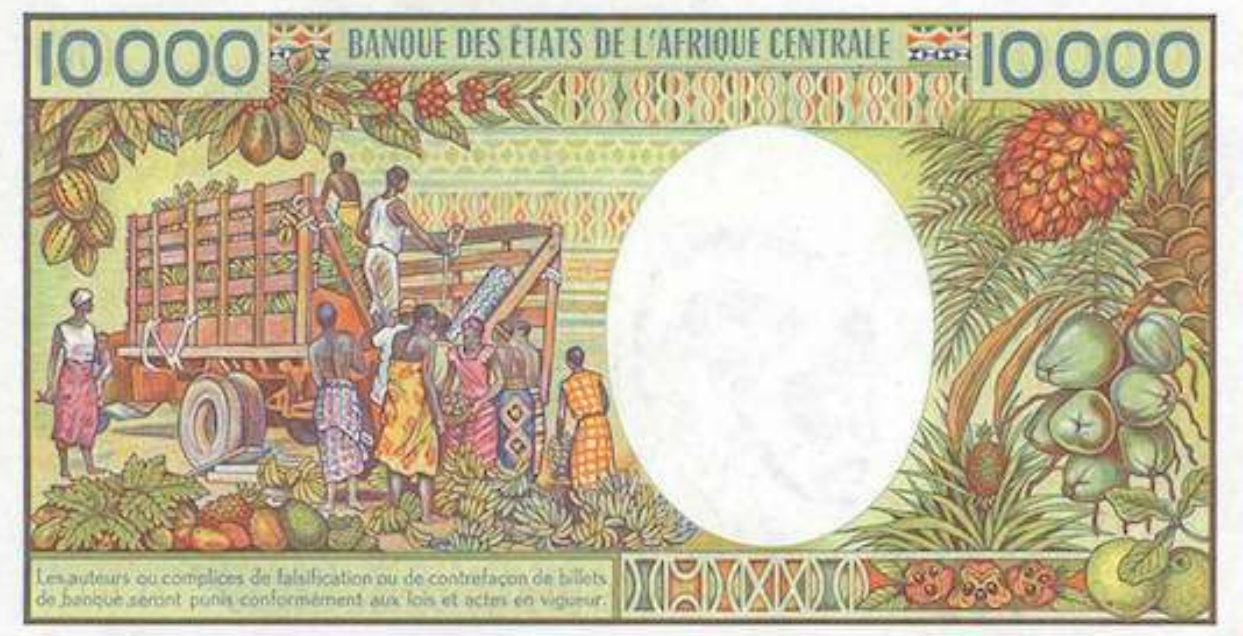

10 000F, 92x169mm, 1982, BEAC, revers

(c) GB Numismatique Paris 
Le revers de la coupure de $10000 \mathrm{~F}$ ci-dessus présente une scène où des hommes déchargent des produits frais d'une semi-remorque, tandis que les femmes les récupèrent et les rangent sur le sol. Cette pratique très courante est le domaine des commerçantes au Cameroun. Il s'agit de revendeuses qui, dès l'aube, attendent l'arrivée des denrées alimentaires qu'elles écoulent tout au long de la journée dans les marchés ou des points de vente dans les quartiers. La force musculaire des hommes est affichée ici dans le transport et la décharge des vivres. Tandis que les rôles des femmes, mères et commerçantes, sont valorisés.

Image 24. Femme portant un fardeau

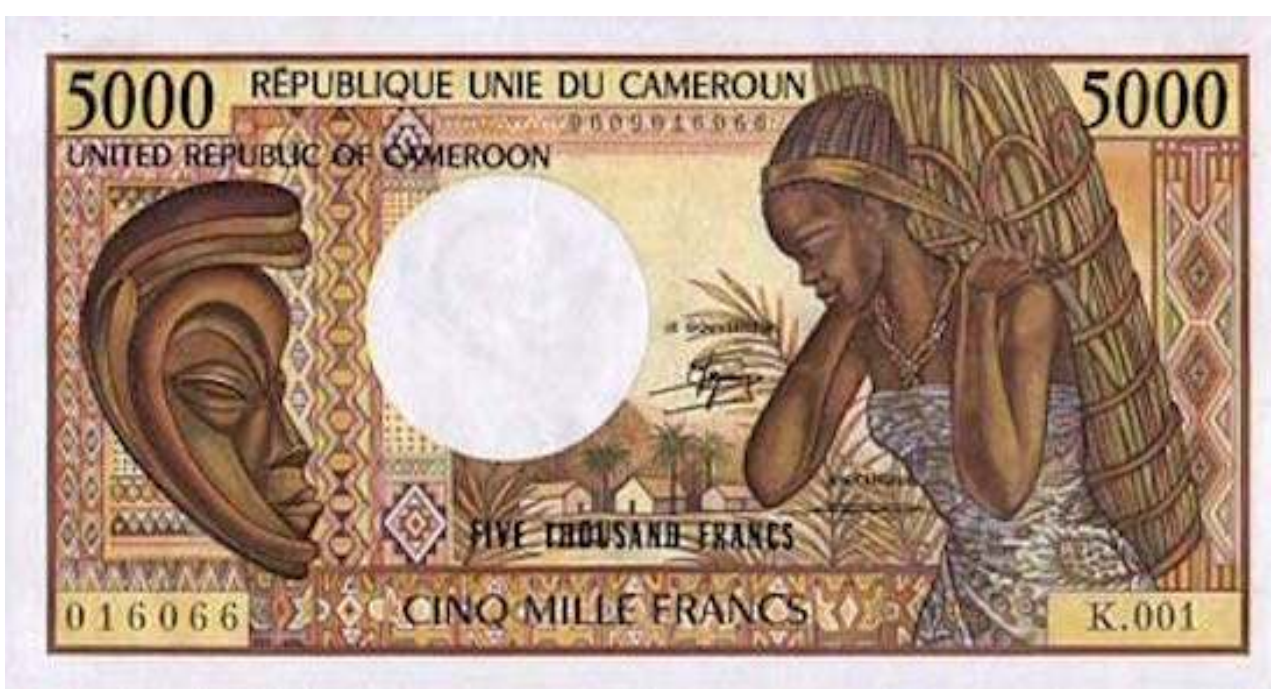

5 000F, 36x162mm, 1982, BEAC, avers

(c)Multicollect.net

Image 25. Homme conduisant un engin agricole

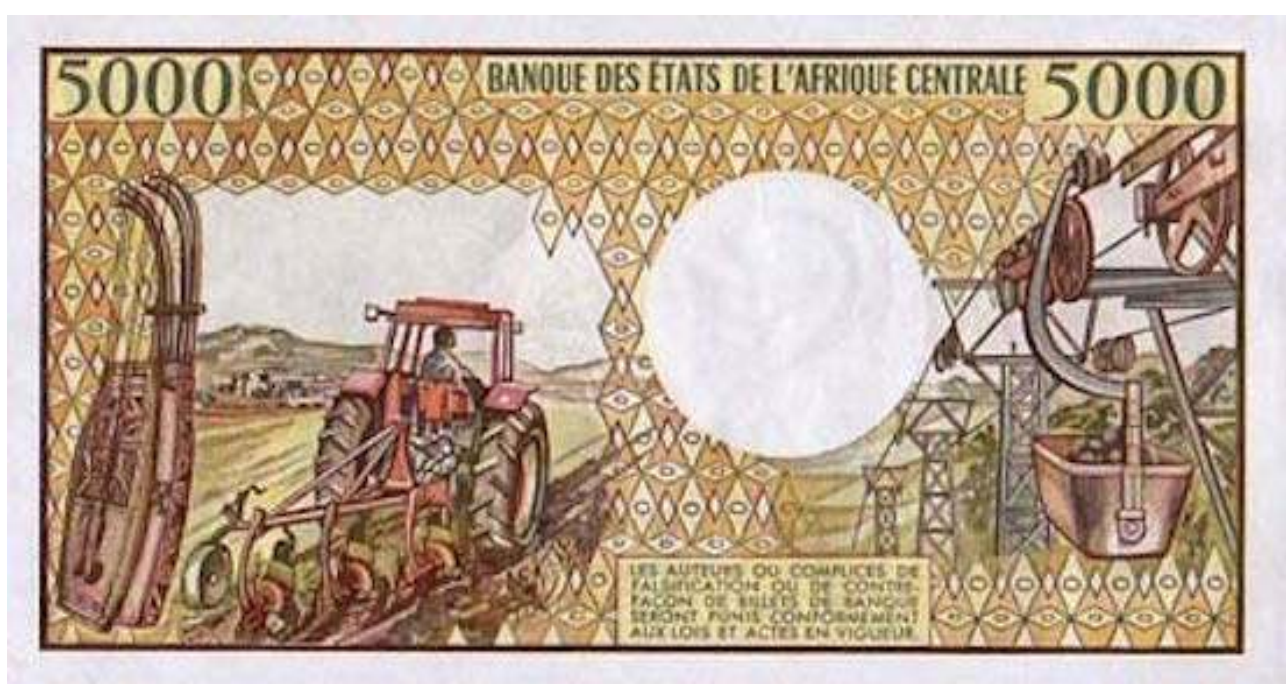

5 000F, 36x162mm, 1982, BEAC, revers

(c)Multicollect.net

L'avers de cette coupure de 1982 présente une jeune femme, aux yeux fermées, portant une hotte de feuilles de raphia sur le dos et soutenue par sa tête et ses mains (image 
24). Cette façon de transporter un fardeau est très répandue à l'Ouest du Cameroun (Champaud, 1983). Ces feuilles servent à fabriquer les balais et les toits de chaume. L'aspect esthétique de la femme disparaît au profit de son travail, qui joue un rôle important dans le développement du pays. Mais sa peine est mise en exergue par le fait qu'elle est montrée les yeux fermés. C'est le quotidien de ces femmes de transporter de lourdes charges de bois, de raphia et de produits agricoles sur le dos ou sur la tête.

Le revers de ce billet de 5 000F émis 1972 présente comme un des deux motifs principaux, un engin conduit par un homme qui cultive une parcelle (image 25). Ici, l'homme aidé par la machine, opère sur des vastes surfaces.

Image 26. Homme sculptant un masque

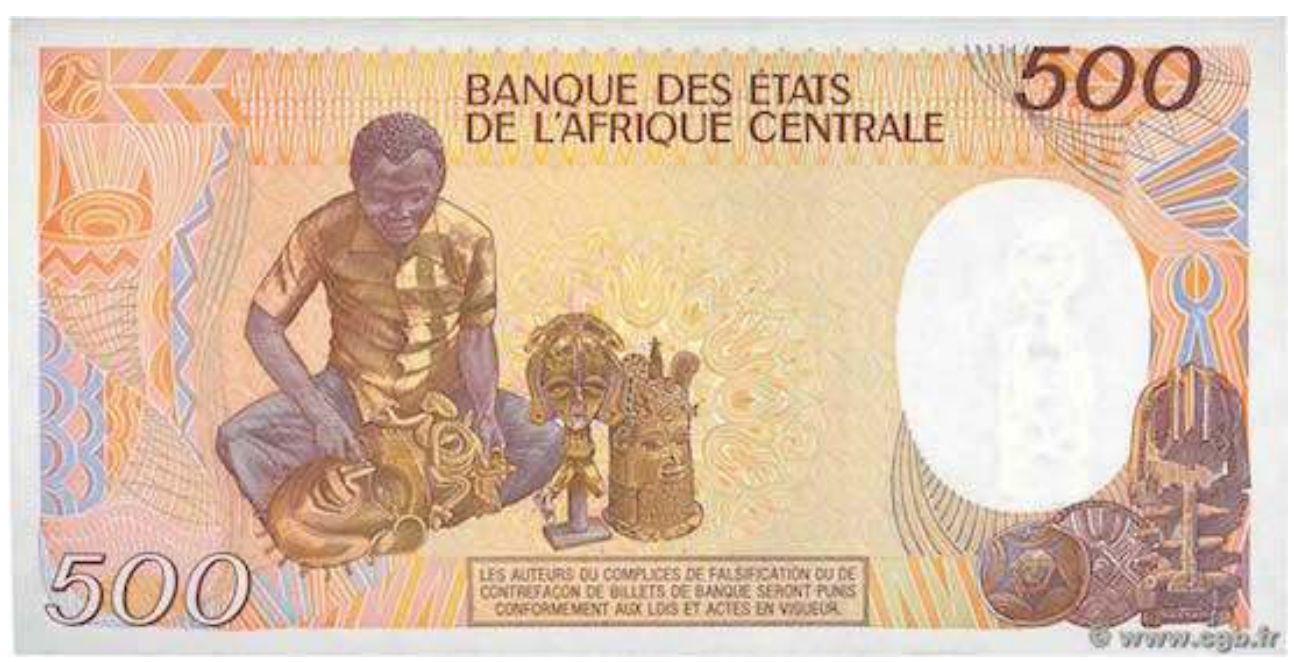

500F, 76x150mm, 1982, BEAC, revers

(C)CGB Numismatique Paris

Sur ce revers du billet de 500F ci-dessus émis en 1982, figure un homme entrain de sculpter un masque, le «masque bamoun", comportant une tête d'un homme couronnée d'un serpent à deux têtes. Les femmes artistes n'apparaissent pas sur les monnaies, pourtant elles existent, mais la sculpture était par le passé, au Cameroun, étroitement liée au pouvoir; les rois et les notables sculptaient eux-mêmes les œuvres (Perrois, 1994). De fait, ce travail ainsi que la commercialisation de l'art, reste, encore aujourd'hui, majoritairement un domaine attribué aux hommes. 
Image 27. Berger abreuvant son troupeau
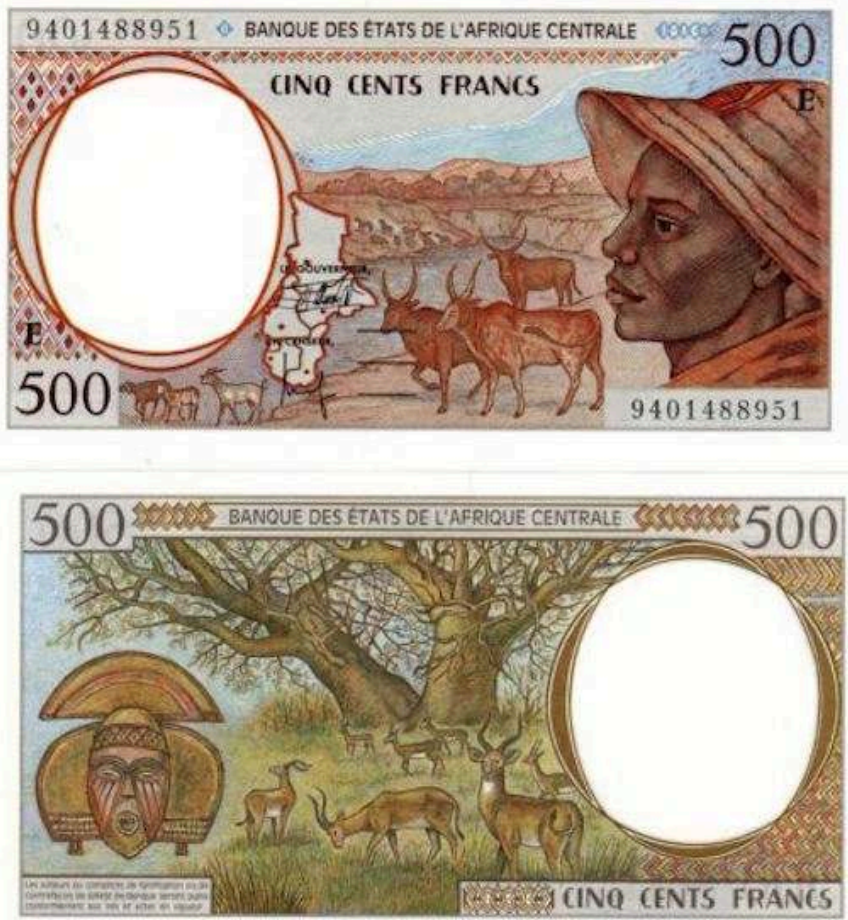

500F, 75x139mm, 1992, BEAC, avers

(C)Multicollect.net

42 Le billet de 500F de 1992 met encore un homme à l'honneur avec le portrait, de profil, d'un berger venu abreuver son bétail. Les cases sont visibles dans le champ du billet mais aucune femme n'est présente pendant cette activité. Pourtant, les femmes y participent en trayant quotidiennement le lait des vaches pour la commercialisation, la consommation ou la transformation.

Image 28. Récolte de café

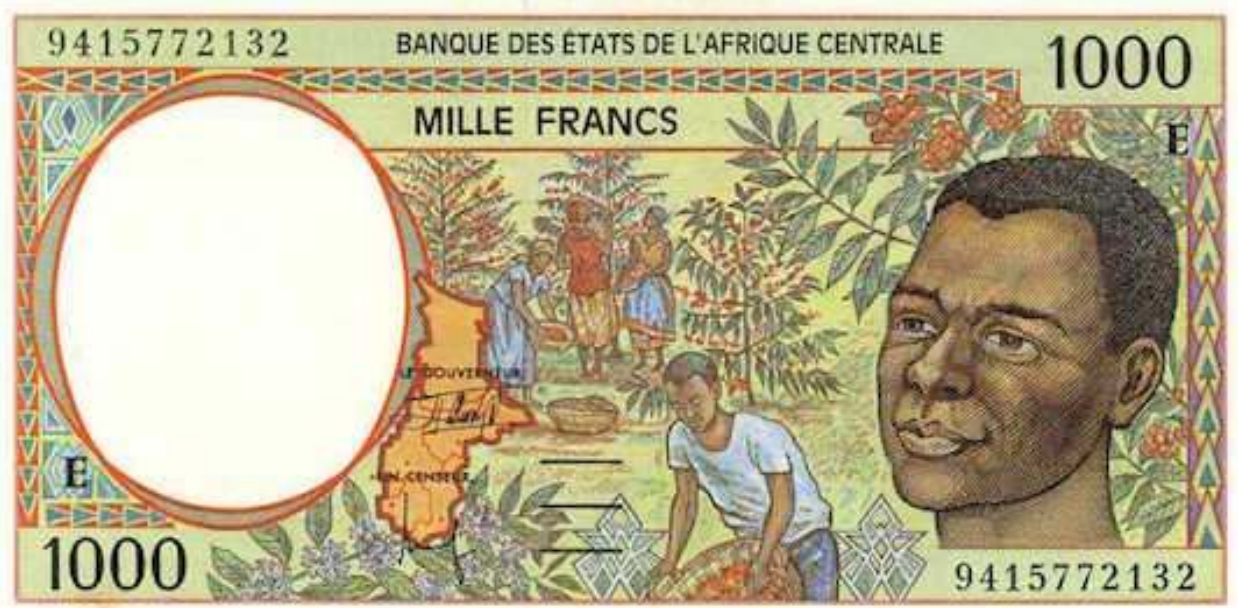

1 000F, 75x148mm, 1992, BEAC, avers

(C)Multicollect.net 
Image 29. Évacuation des grumes

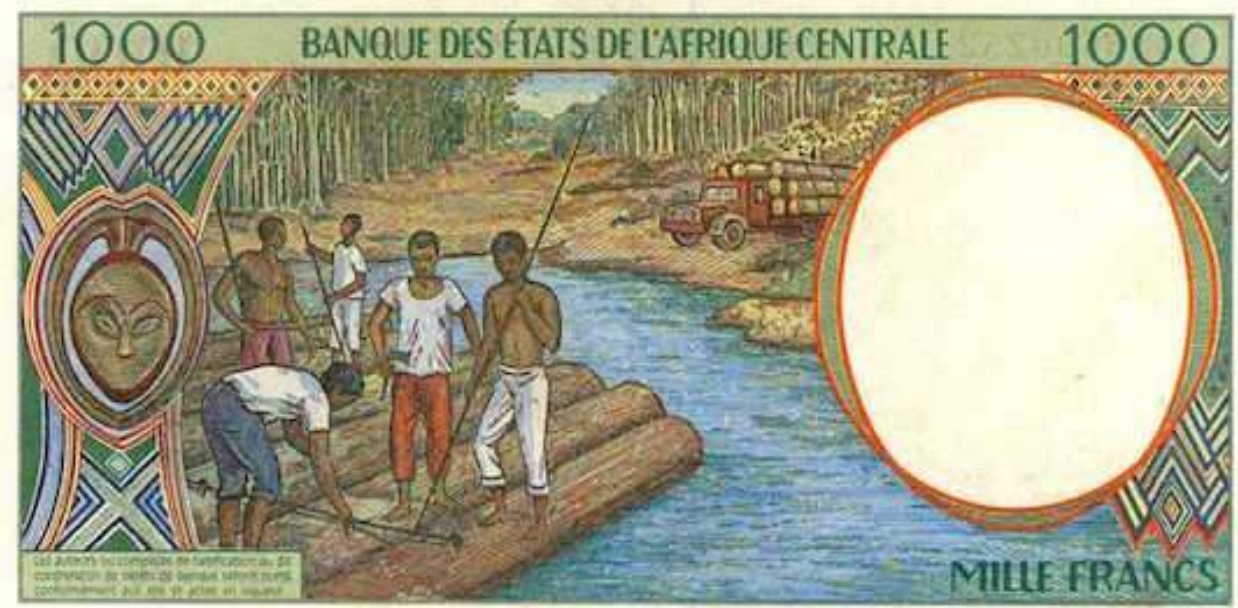

1 000F, 75x148mm, 1992, BEAC, revers

(C)Multicollect.net

43 Encore appelé «billet vert ", cette coupure de 1000F montre à l'avers ci-dessus une récolte de café : trois femmes entrain de cueillir du café et un homme entrain de vider un panier (image 26). L'homme souvent, en plus de la cueillette, est chargé de transporter le panier plein et d'aller le vider tandis que les femmes continuent la cueillette sur place. L'apport de la force physique est encore mis en exergue et le dur labeur de l'homme s'illustre sur les traits attristés du portrait mis au premier plan. Sur le revers, le travail de l'homme est encore mis en avant à travers l'exploitation forestière. Une semi-remorque transportant des billes de bois sort de la forêt et des hommes sont stationnés sur des billes de bois jetées dans le cours d'eau. Aucune femme n'intervient à ce niveau alors qu'elles peuvent participer au développement de ce secteur (Lema Ngono Nyom Pom, 2007).

L'activité commune des femmes et des hommes dans l'agriculture persiste, mais les représentations, qui se limitent à la récolte, ne permettent pas de voir clairement les implications de chaque sexe. Le fait que les hommes soient impliqués dans le transport des produits récoltés (images 23 et 28 ) marque une fois de plus une différence de traitement iconographique suivant le genre. 


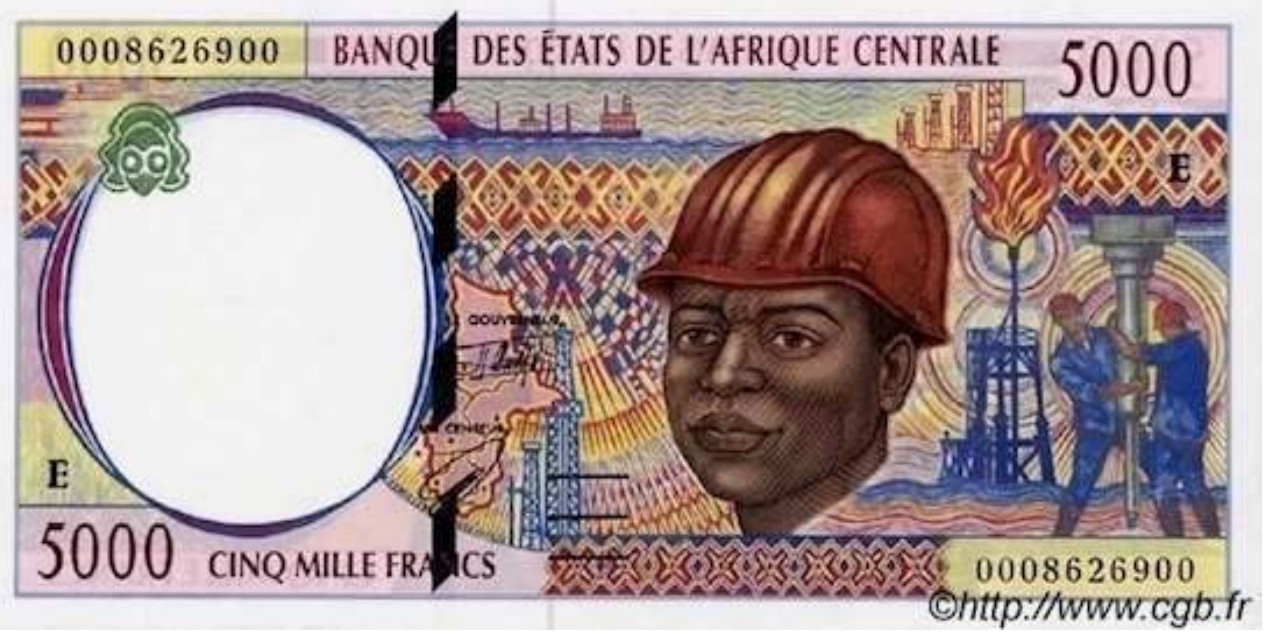

5000F, 80x161mm, type 1992, BEAC, avers

(c) GB Numismatique Paris

Image 31. Séchage de coton par les hommes et les femmes

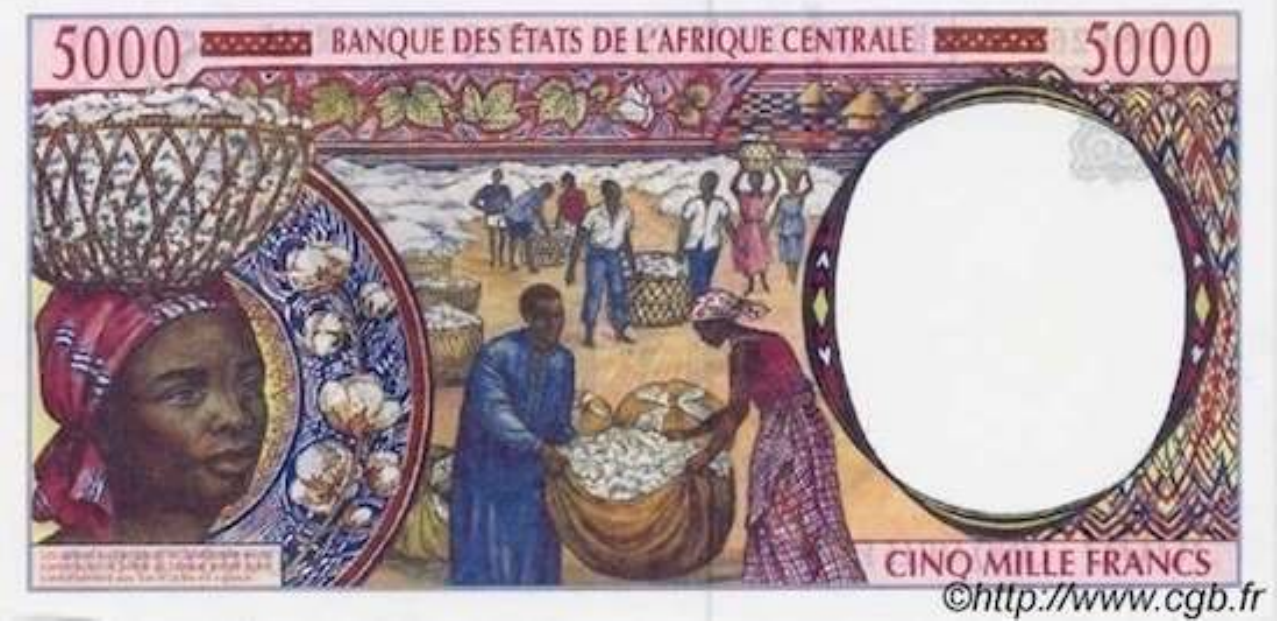

80x161mm, type 1992, BEAC, revers

(C)CGB Numismatique Paris

En 1992, la coupure de 5000F émise par la BEAC porte à l'avers, la représentation d'un ouvrier dans une compagnie pétrolière (image 28). C'est ce qui est illustré dans le champ du billet avec des hommes certainement en pleine prospection pétrolière. Il s'agit d'une activité majoritairement masculine. Les différentes étapes d'exploitation nécessitent de la force musculaire et une prise de risque. Les femmes, elles, font de l'agriculture et du commerce comme le démontre l'image secondaire au revers.

46 Il présente en effet le séchage du coton dans un village sahélien où hommes et femmes participent ensemble au transport du produit. À gauche, la représentation d'une femme portant sur la tête un panier de coton témoigne de l'importance du travail féminin dans la culture et le processus entier de production de cette denrée. Matière première d'exportation cultivée en grande quantité, la culture et la transformation du coton nécessite une main d'œuvre abondante et mixte. 


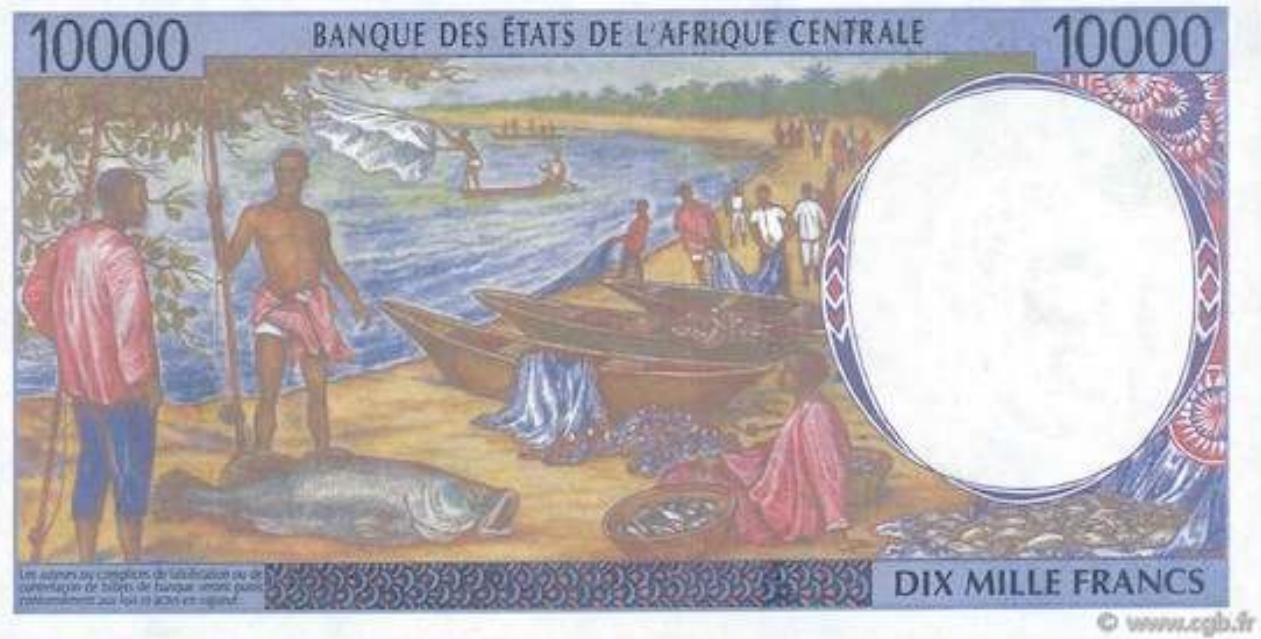

10000 F, 80x161mm, 1992, BEAC, revers

(c) GB Numismatique Paris

Le revers de la coupure de 10000 Francs ci-dessus présente une scène de pêche artisanale essentiellement masculine, faite au moyen des pirogues. Le butin de cette pêche, matérialisé par un très gros poisson, peut traduire l'essor de la pêche artisanale comme source de richesse de ces populations. Les femmes apparaissent en arrière-plan, en chemin pour le lieu de dépôt des produits de la pêche. La description physique du pécheur à moitié nu permet d'insister visuellement sur l'effort nécessité par cette activité et donc une valorisation de la force musculaire virile.

Image 33. Femme près des cases

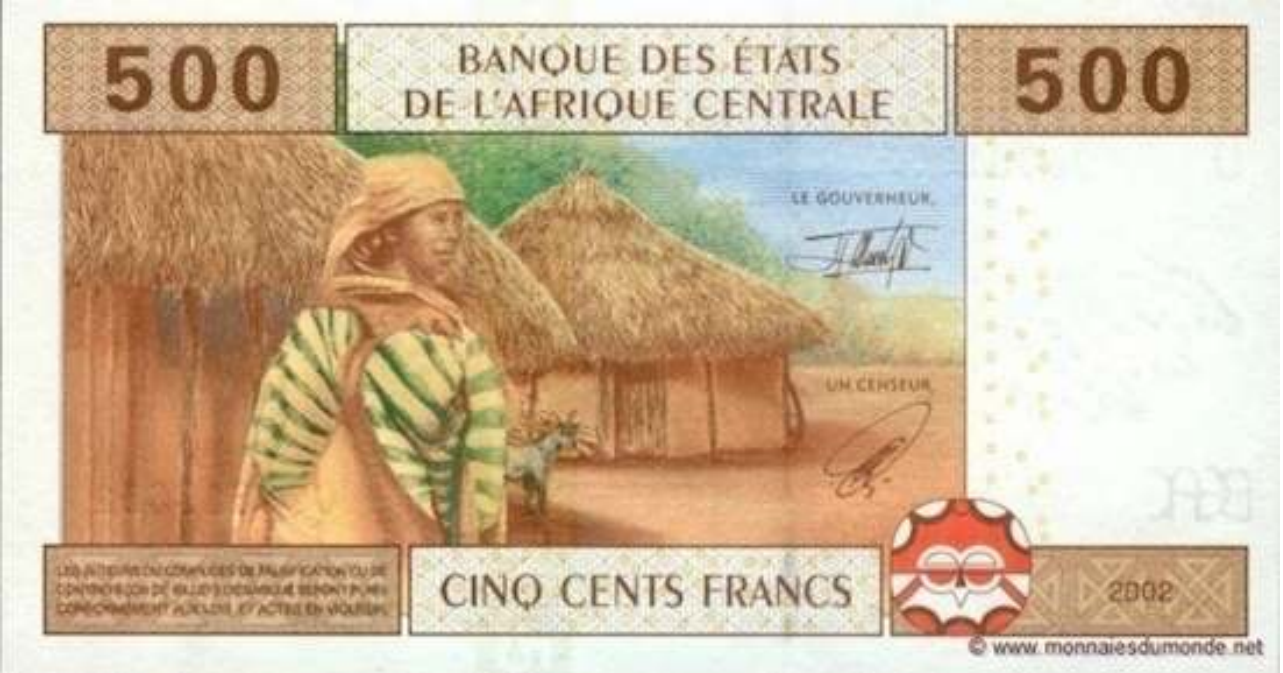

70x130mm, 2002, BEAC, avers

(c)monnaiesdumonde.net-avec autorisation de David NISON 


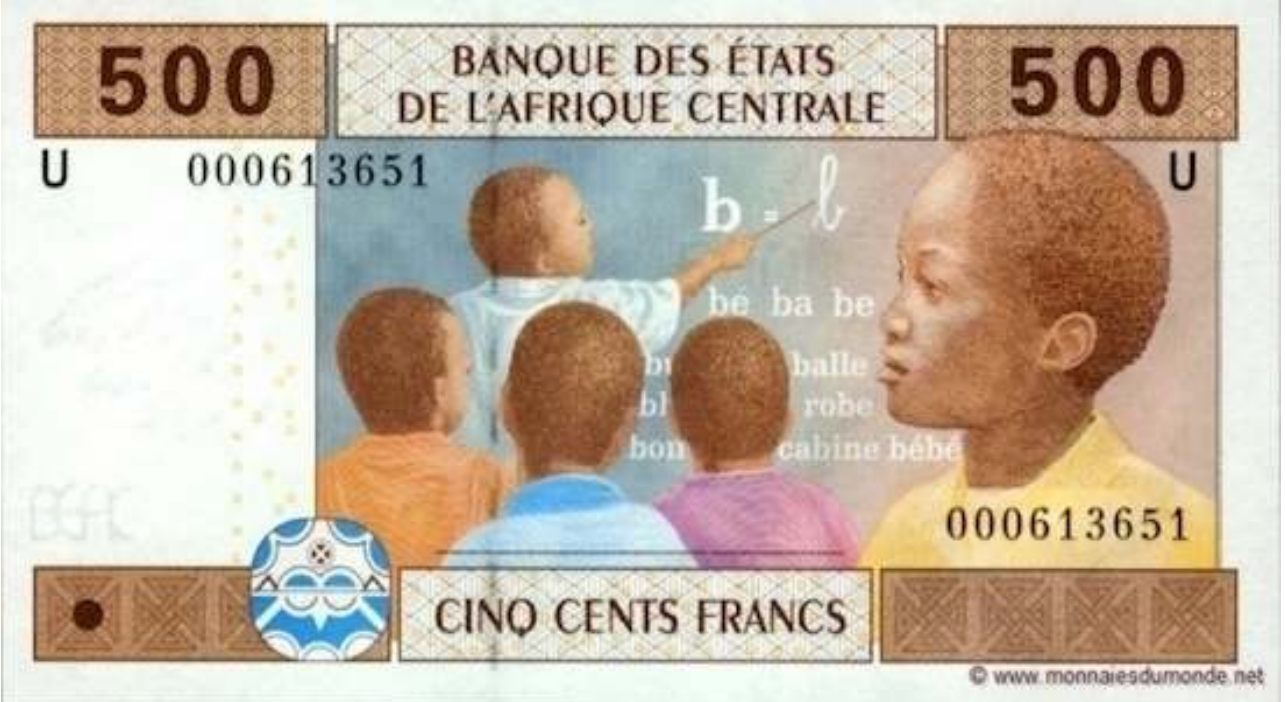

70x130mm, 2002, BEAC, revers

(C)monnaiesdumonde.net-avec autorisation de David NISON

La coupure de 500 Francs ci-dessus émise récemment en 2002 présente à l'avers une femme dans une concession (image 31). Il s'agit d'une femme peule qui est assignée généralement à rester à la maison pour assurer les tâches quotidiennes de son entretien. Contrairement aux images relatives au travail des hommes, l'absence de détails ne permet pas de saisir le véritable rôle social de la femme camerounaise parmi lesquels la procréation, l'éducation et la nutrition. Le revers montre, quant à lui, des élèves dans une salle de classe (image 33). Aucune fille n'est présente. Pourtant, l'instruction des jeunes filles au Cameroun est un défi après les indépendances. Elle progresse à partir de 1990 (en moyenne $45 \%$ au primaire et au secondaire) en Afrique centrale où elle figure en tête des préoccupations après les années 1990 (Marmoz, 2006). La tendance se renverse aujourd'hui. De plus en plus nombreuses dans les salles de classes, les filles y sont plus représentées que les garçons. 


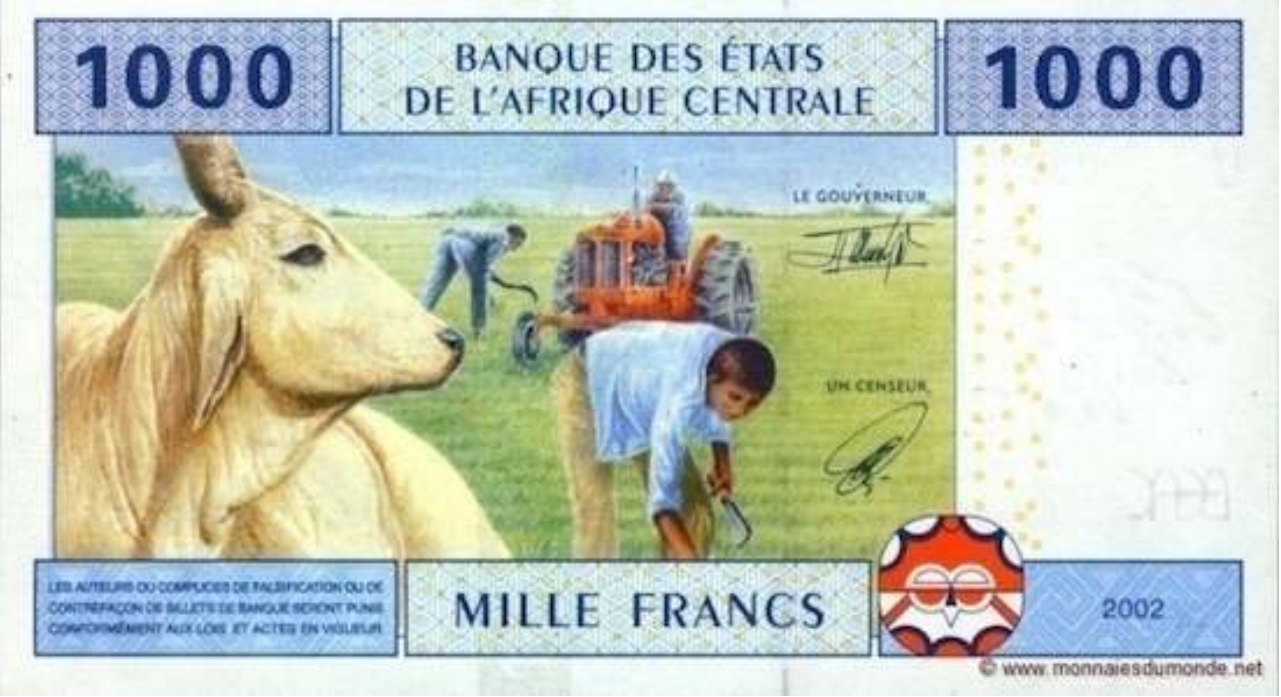

$1000 \mathrm{~F}, 75 \times 135 \mathrm{~mm}, 2002$, BEAC, avers

(C)monnaiesdumonde.net-avec autorisation de David NISON

Image 36. Homme dans un tracteur

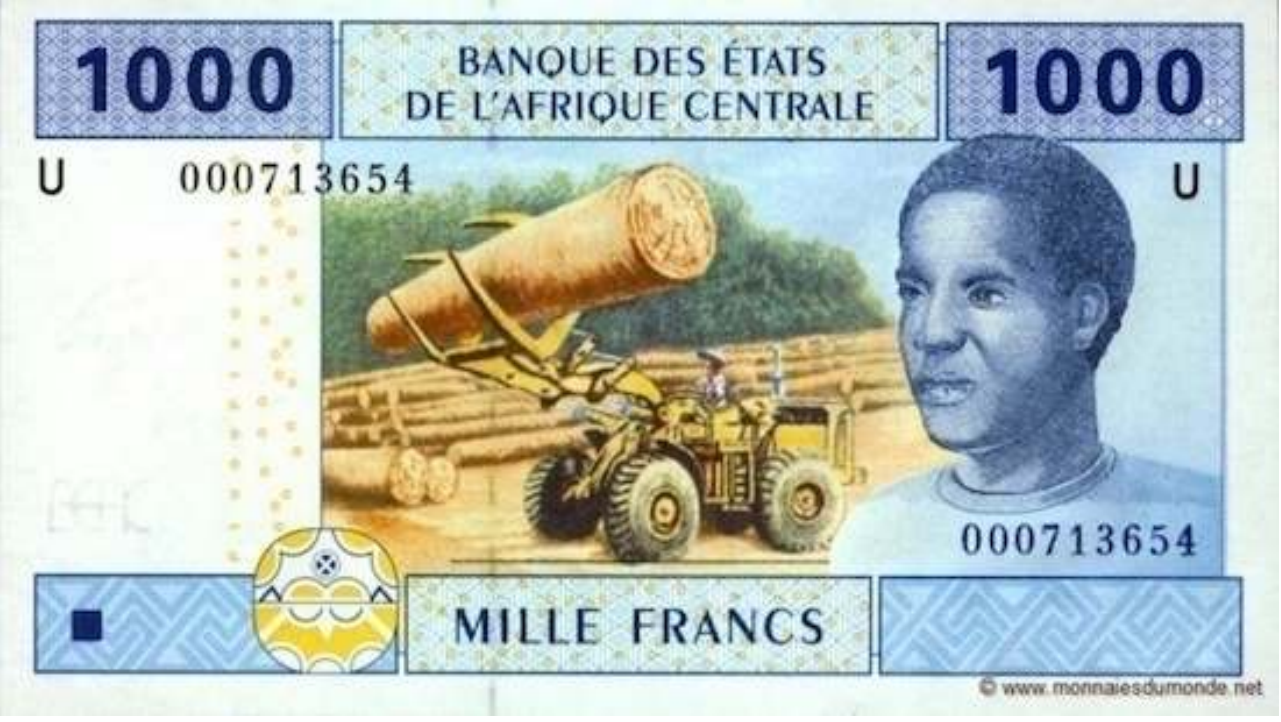

$1000 \mathrm{~F}, 75 \times 135 \mathrm{~mm}, 2002$, BEAC, revers

(C)monnaiesdumonde.net-avec autorisation de David NISON

La face de ce billet (image 34) illustre une ferme et le motif principal est une vache. Les travailleurs, hommes, utilisent des "coupe-coupe", des machettes utilisées couramment au Cameroun, tandis qu'un autre conduit le tracteur. Les hommes pratiquent de moins en moins le travail manuel pour des grandes surfaces. Sur le revers (image 35), c'est la représentation d'un jeune homme au regard inquiet qui est représenté. Dans le champ, est illustré un engin de transport du bois conduit par un homme.

Dans cette dernière génération de billets, l'homme joue toujours un rôle plus important dans l'organisation du travail par rapport à la femme qui est au second plan. Les 
travaux champêtres et le commerce seraient ses domaines de prédilection. L'industrie, la formation et même l'art seraient réservés aux hommes. Cela ne correspond pourtant pas à la réalité des pays membres de la CEMAC. Les femmes participent à ces activités, parfois en petit nombre (industrie) mais elles n'en sont donc pas absentes (MINFPROFF, 2012). Les jeunes filles vont à l'école et participent à la formation. Les femmes travaillent dans les foyers et cette forme de travail est totalement mise à part (deux images). Il s'agit là d'une image genrée par sa construction socio-sexuée des rôles suivant le sexe et l'âge des personnages cloisonnés dans leurs activités suivant une attribution.

51 En définitive, cette tentative d'analyse iconographique des monnaies coloniales puis postcoloniales, particulièrement du Franc CFA en circulation au Cameroun depuis 1945 a permis de comprendre que l'effort de division des tâches n'est pas représentatif de la société camerounaise uniquement, mais une vision globale des possessions françaises d'Afrique Centrale, d'Outre-Mer. Bien que le Cameroun n'ait pas été une colonie française, il a bénéficié des mêmes images monétaires que l'ensemble de son empire colonial, malgré ses titres: «territoire sous mandat», "territoire sous tutelle " et « République ». Peut-être en raison du coût élevé de production de monnaies, la France n'a guère pris le soin de lui attribuer une iconographie particulière.

Ces représentations stéréotypées du genre et du travail dans les images monétaires au Cameroun sont donc le fruit de l'image que la France s'est faite des Africains pendant et après la colonisation. Les images du travail, majoritaires parmi les thèmes développés, ne permettent de reconnaître que la part des hommes, sacrifiés à la réussite de colonisation française. Elles montrent la puissance de la France dans ses possessions malgré les ravages de la guerre et justifient sa présence dans ses possessions coloniales, y compris pendant une période tendue de la libération. Cela passe par l'exhibition des matières premières dont la France a besoin et qui se cultivent mieux dans ces territoires et de la main d'œuvre qui n'est toujours qu'africaine. Le pouvoir et le gain orientent ainsi cette iconographie, plongeant dans l'ombre les valeurs traditionnelles des peuples représentés.

Enfin, la catégorisation homme/femme ne semble pas être une grande préoccupation de l'émetteur qui s'intéresse plus aux produits et richesses locales qu'aux habitante's des territoires. De même, les images telles que représentées, obéissent à une répartition des tâches avec pour point de mesure la force physique. Parmi les travaux intéressants pour la France au Cameroun, ceux qui nécessitent plus de force sont attribués aux hommes. Cette méthode de division des tâches rappelle l'organisation du travail à l'ère de la traite négrière, puis celui de l'économie coloniale ou de traite où la priorité était le rendement et non les personnes (Badouin, 1967). Dans la réalité, selon les peuples et leurs religions, les tâches sont plus diversifiées et la frontière entre les travaux des hommes et ceux des femmes n'est pas étanche. Cette iconographie s'oppose à celle des anciennes colonies d'Afrique de l'Ouest dont les images monétaires semblent plus variées culturellement et à l'iconographie monétaire de la France où les thèmes relatifs au travail semblent passéistes. 


\section{BIBLIOGRAPHIE}

Franqueville A. (1985), « l'offre paysanne en produits vivriers dans le sud du Cameroun », Nourrir les villes en Afrique Subsaharienne, Paris, l'Harmattan, p. 122-135.

Barthes R. (1964), « Rhétorique de l'image », Communication, « Recherches sémiologiques », $n^{\circ} 4$, p. $40-51$.

Badouin R. (1967), « Où en est la réforme de l'économie de traite en Afrique noire? », Tiers-Monde, tome 8, n 32, L'Espagne à l'heure du développement, p. 1209-1216.

Barbier J.C. (dir), 1985, Femmes du Cameroun. Mères pacifiques, femmes rebelles, ORSTOM, KARTHALA. Blanchard P. (2001), « La représentation de l'indigène dans les affiches de propagande coloniale : entre concept républicain, fiction phobique et discours racialisant ", Hermès, n 30, p. 149-168. Boutrais J. (1994), « Les foulbés de l'Adamaoua et l'élevage : de l'idéologie pastorale à la pluriactivité », Cahiers d'études africaines, p. 175-196.

Boutrais J. (1999), « Journées de bergers au Nord-Cameroun », Les temps du Sahel : en hommage à Edmond Bernus, Paris, IRD, p. 55-80.

Caire G. (2002), « Les billets comme mass médias : de la "Fortune" de 1803 aux "portails, fenêtres et ponts" de 2002 », Économies et Sociétés, Tome XXXVI, n 1, p. 35-54.

Champaud, (1983), Villes et campagnes du cameroun de l'Ouest, Paris, O.R.S.T.O.M, p.300.

Deganendji G. (2006), « Monnaie et échanges en Afrique centrale zone franc CFA XIXe-XXe siècles ", Thèse de Doctorat, Université de Yaoundé I.

Duprat A. (2007), Images et Histoire: Outils et méthodes d'analyse des documents iconographiques, Paris, Belin.

Dupré M. (2001), « Figures de l'argent », La Voix du regard, n 14, p. 23-30.

Gérardin H. (1989), La zone franc. Tome 1 : Histoire et institution, Paris, L'Harmattan.

Gervereau L. (1994), Voir, comprendre, analyser les images, La Découverte, Paris.

Guétat-Bernard H. (2011), « Culture du café et transformations des rapports de genre en pays bamiléké au Cameroun », Du grain à moudre. Genre et développement rural et alimentaire, Genève, IUED, p.167-188.

Goerg O. (1997), «Femmes africaines et politique : les colonisées au féminin en Afrique occidentale », Clio. Histoire, femmes et sociétés, [En ligne], n 6, mis en ligne le 01 janvier 2005, consulté le 15 mai 2018. URL : http://clio.revues.org/378

Kembe Milolo P. (1986), «L'image de la femme noire chez les romancières d'Afrique noire francophone », Thèse de Doctorat, Université de Fribourg.

Lema Ngono Nyom Pom D. (2007), « Intégrer les questions de genre dans le secteur forestier en Afrique Cameroun », Organisation des Nations Unies pour l'Alimentation et l'Agriculture, [En ligne], mis en ligne le 18 octobre 2007, consulté le 03 janvier 2018. URL : www.agris.fao.rog

Fonkoua P. (2006), « Femme et Education au Cameroun : de la logique d'un état à l'état d'une logique », La scolarisation des filles au Cameroun : jalons, repères et perspectives, Paris, L'Harmattan, p. 5-6. 
Martin J-Y. (1968), Les Matakam du Nord-Cameroun : dynamismes sociaux et problèmes de modernisation, Thèse de Doctorat, Université de Yaoundé.

Mezzana D. et Anglana T. W. (2002), « Une représentation, source de ségrégation : la construction de l'Afrique comme un monde à part », [En ligne], mis en ligne le 20 mai 2002, consulté le 02 mars 2013. URL : http://www.africansocieties.org/n1/fr_paginaarticolo4.htm

MINFPROFF (2012), « Femmes et hommes au Cameroun en 2012. Une analyse situationnelle du progrès en matière de genre ", Institut National de la Statistique, Yaoundé. [En ligne], mis en ligne le 28 février 2012, consulté le 29 mai 2018. URL :http://www.statistics-cameroon.org/downloads/ JIF/MINPROFF_Femmes_Hommes_Cameroun02_2012.pdf

Monpas G. (2006), « Recherches sur l'iconographie romaines de la victoire des origines à la fin des Antonins : Etude numismatique », Thèse de Doctorat, Université de Genève.

Peirce C. S. (1978), Écrits sur le signe, rassemblés traduits et commentés par Gérard Deledalle, Paris, Le Seuil.

Perez C. (1985), «Images monétaires et pratiques sémiologiques », Dialogues d'histoire ancienne, v. 11, p.110-140.

Perrois L. (1994). « Sculpteurs et notables (Ouest-Cameroun), Créer en Afrique », 2e colloque européen sur les arts d'Afrique noire, p.115-120.

Picard O. (1991), «Images des dieux sur les monnaies grecques », Mélanges de l'Ecole française de Rome. Antiquité, Volume 103, n 103-1, p. 223-233.

Renaud É. (1899), « Les banques coloniales », Thèse de Doctorat, Université de Poitiers.

REFACOF (2014), «État des lieux relatif au genre dans le déboisement et la dégradation des forets au Cameroun », Réseau des Femmes africaines pour la

Gestion Communautaire des Forets, [En ligne], mis en ligne le 03 octobre 2014, consulté le 28 janvier 2018. URL : www.refacof.org

Reuter P. (1950), Les francs coloniaux, Paris, Dalloz.

Sah, L. (2008), Femmes bamiléké au maquis. Cameroun, 1955-1971, Paris, L'Harmattan.

Schomas H. (2011), « Les images monétaires des peuples gaulois : figures primitives ou expression d'une société en mutation ? : l'exemple des Arvernes, Bituriges, Carnutes, Eduens, Meldes, Parisii, Sénons et Séquanes ", Thèse de Doctorat, Université de Bourgogne.

Vincent J.-F. (1979), « Place et pouvoir de la femme dans les montagnes mofu (Nord-Cameroun) », Cahiers d'études africaines, Vol. XIX, p. 225-251.

\section{NOTES}

1. Plusieurs autres thèmes apparaissent dans l'iconographie monétaire au Cameroun: le colonialisme, l'économie, l'esthétique, la politique, l'art, etc.

2. Lire à ce propos Goerg O. (1997), «Femmes africaines et politique : les colonisées au féminin en Afrique occidentale ", Clio. Histoire, femmes et sociétés, [En ligne], $\mathrm{n}^{\circ} 6$, mis en ligne le 01 janvier 2005, consulté le 15 mai 2018. URL : http://clio.revues.org/378

3. Les femmes n'étaient pas uniquement des« instruments " de travail, elles étaient aussi une source de plaisir sexuel. Lire à ce propos Jeurissen L. (2003), «Colonisation et mise en corps de la féminité noire : cas de l'ancien Congo Belge », séminaire FER. ULg 2002-2003, Cycle femme et 
corps. [En ligne], mis en ligne le 08 mai 2003, consulté le 29 mai 2018. URL: http:// www.congoforum.be/upldocs/Jeurissen.pdf

4. La présence d'images liées au travail permettait de justifier l'action coloniale et de glorifier l'effort des occidentaux dans la quête des ressources dans les nouveaux mondes.

5. Cette observation est le fruit de notre travail de Master intitulé «L'iconographie monétaire au Cameroun. Approche historique », soutenu à l'Université de Ngaoundéré en 2012.

6. La zone franc est non seulement un espace monétaire, mais aussi un espace économique et culturel, créé par la France en 1939 pour « isoler » son empire colonial du marché international après la grande crise de 1929. Il s'agissait de mobiliser et contrôler incessamment ses possessions coloniales dans une subordination monétaire, même après les indépendances. Elle se réclame d'entretenir des relations économiques basées sur une stabilité monétaire. Lire à propos Banque de France, 2002, «La zone franc ", Note d'information, $\mathrm{n}^{\circ}$ 127, 12 pages.

7. Les billets filmés étaient de mauvaise qualité, en termes de visibilité. Nous avons donc opté pour l'obtention des images par des sites de collectionneurs et vendeurs de monnaies à qui nous avons envoyé des demandes d'autorisation d'utilisation d'images.

8. Lire à propos de la nudité : Martin, J.-Y. (1968), Les Matakam du Nord-Cameroun: dynamismes sociaux et problèmes de modernisation, Thèse de Doctorat, Université de Yaoundé.

9. Un aperçu du regard colonial sur la femme indigène est fait par Quillet, L. (2014), « La femme indigène, fantasme colonial », [En ligne], mis en ligne le 29 août 2014, consulté le 15 mai 2018. URL : http://madame.lefigaro.fr/art-de-vivre/femme-indigene-fantasme-colonial-290814-901383 10. Lire Yann Le Bihan, (2007), «La "femme noire" dans L'Écho des Savanes », Revue des Sciences Sociales, $n^{\circ} 38$, p.140-149. [En ligne], consulté le 29-05-2018. URL : http://www.revue-des-sciencessociales.com/pdf/rss38-lebihan.pdf

11. Lire à ce propos Sah, L. (2008), Femmes bamiléké au maquis. Cameroun, 1955-1971, Paris, l'Harmattan.

12. Le France CFA est émis jusqu'à l'heure actuelle par la France; les artistes et graveurs des maquettes de ses illustrations sont choisi par la France.

13. Ahmadou Babatoura Ahidjo (1924-1989) est le premier président de la République du Cameroun. Originaire du Nord-Cameroun, il intègre l'administration française et occupe plusieurs postes dans le gouvernement avant de proclamer l'indépendance le 1er janvier 1960 ; il est élu le 05 mai 1960 comme premier président de la République du Cameroun. En 1961, après la réunification, le Cameroun pris le nom de République Fédérale du Cameroun. Il instaure un régime autoritaire jusqu'à sa démission en 1982.

\section{RÉSUMÉS}

Cet article s'inscrit dans une perspective d'analyse d'iconographie monétaire et tente d'interroger les stéréotypes genrés de la place de la femme dans le monde du travail. Mises en circulation par la France, ces images restituent difficilement l'essence de la notion de travail telle que vécue par les différents peuples du Cameroun aux valeurs culturelles et religieuses plurielles. Elles illustrent, de façon globale, le regard du colonisateur sur les travaux des hommes et des femmes dans le processus colonial puis postcolonial, lequel est le principal pourvoyeur d'activités professionnels. Ainsi, elles ne montrent pas l'organisation sociale originelle qui était à l'image de la tradition orale de ces peuples aux essences culturelles quasi communes, mais permettent d'apprécier la participation de ceux-ci à la construction de la richesse de la métropole à travers 
l'exhibition des matières premières. À la veille de l'indépendance, des efforts de localisation sont faits et, progressivement, les images interpellant les réalités sociales camerounaises apparaissent.

This paper intends to examine gender stereotypes in the representations of women labouring, as they are depicted in monetary iconography. Those currency images are circulated by French government: they do not exactly show work as experienced by Cameroon people, whom cultural and religious values vary from on community to another. They generally demonstrate how a dominant culture perceive working men and women, within a colonial, and then postcolonial, systemic process, which is, as it is, the main work-provider. Thus, those banknotes do not show the original social organisation that was in the image of an oral tradition, shared by almost same cultural populations. Through the iconographic exhibition of numerous raw materials, they allow to see how people participated in building Metropolitan France's wealth. On the verge of Cameroon Independence, the images are progressively more specific about geographic localisation and begin to let appear more of the gender and social reality of the country.

\section{INDEX}

Keywords : money, iconography, gender, stereotype, work

Mots-clés : iconographie monétaire, genre, stéréotype, travail

\section{AUTEUR}

\section{JOCELINE CLARISSE MAFOSSI}

Mafossi Joceline Clarisse est une Camerounaise et titulaire d'un Master en Histoire obtenu à l'université de Ngaoundéré au Cameroun. Elle est doctorante dans la même université où elle achève une thèse intitulée « Iconographie monétaire au Cameroun de 1916 à 2006 ». 